\title{
BIG IMAGE OF GALOIS REPRESENTATIONS AND CONGRUENCE IDEALS
}

\author{
HARUZO HIDA AND JACQUES TILOUINE
}

\section{Contents}

1. Introduction 1

2. Galois representations associated to Siegel modular forms 2

3. Fullness of the image for Galois representations in $\operatorname{GSp}(4) \quad 3$

3.1. Irreducibility and open image 3

3.2. Connected components of the image of Galois 5

3.3. Connected semi-simple subgroups of GSp(4) 5

3.4. $\mathbb{Z}_{p}$-Fullness for GSp(4) and its subgroups 6

3.5. Pink's Lie algebra theory 7

3.6. $\Lambda$-fullness for the endoscopic cases 8

3.7. $\mathbb{Z}_{p}\left[\left[S_{1}\right]\right] \oplus \mathbb{Z}_{p}\left[\left[S_{2}\right]\right]$-fullness in Case $(Y) \quad 10$

4. Fullness of the Galois image in general Spin groups over Iwasawa algebras 11

4.1. Spin groups 11

4.2. Genus $n$ Hida families 11

4.3. $\Lambda_{n}$-fullness $\quad 15$

5. Galois level and Congruence Ideals 18

5.1. Congruence ideals 18

5.2. Comparison between Galois level and congruence ideals 19

References 21

\section{INTRODUCTION}

Let $n \geq 1$; we consider the $n \times n$ antidiagonal unit matrix $s=\left(\delta_{i, n+1-j}\right)_{1 \leq i, j \leq n}$ and the $2 n \times 2 n$ antisymmetric matrix $J=\left(\begin{array}{cc}0 & s \\ -s & 0\end{array}\right)$; we denote by $G=\mathrm{GSp}_{2 n}$ the Chevalley group of symplectic similitude matrices for $J$ and by $B=T N$ its standard Borel consisting of upper triangular matrices in $G$; let $\rho$ be the half-sum of positive roots for $(G, B, T)$ and $\lambda \in X^{*}(T)$ a dominant weight. Let $\pi$ be a cuspidal automorphic representation on $G\left(\mathbf{A}_{\mathbb{Q}}\right)$ of level $M \geq 1$, whose infinity type is in the discrete series, with infinitesimal character $\lambda+\rho$. It occurs in the cohomology of the genus $n$ Siegel variety of level $M$, with coefficients in the local system of highest weight $\lambda$. It implies that there exists a number field $K_{0}$ whose ring of integers $\mathcal{O}_{0}$ contains all Hecke eigenvalues of $\pi$ (for prime-to- $M$ Hecke operators). Let $G_{\mathbb{Q}}=\operatorname{Gal}(\overline{\mathbb{Q}} / \mathbb{Q})$ and $\widehat{G}$ be the dual Chevalley group for $(G, B, T)$; one has $\widehat{G}=\mathrm{GSpin}_{2 n+1}$ (see below); we assume that the compatible system of Galois representations $\rho_{\pi, \iota_{\ell}}: G_{\mathbb{Q}} \rightarrow \widehat{G}\left(\overline{\mathbb{Q}}_{\ell}\right)$ associated to $\pi$ and to embeddings $\iota_{\ell}: \overline{\mathbb{Q}} \hookrightarrow \overline{\mathbb{Q}}_{\ell}$ is constructed (for the moment, it is known for $n=1,2$ ). Let $p \nmid M$ be a rational prime. Assume that $\pi$ is ordinary for $\iota_{p}$; there exists an $n$-variable Hida family passing by $\pi$; more precisely, let $\Lambda_{n}$ be the $n$-variable Iwasawa algebra and $\mathbf{T}^{M}$ be the ordinary prime-to- $M$ Hida-Hecke algebra [MT02], [H02], [Pi12] ; it

Date: August 16, 2014.

The first author is partially supported by the NSF grant: DMS 0753991. The second author is partially supported by the ANR grant: ArShiFo ANR-10-BLAN-0114. 
is finite torsion-free over $\Lambda_{n}$. A Hida family is a $\Lambda_{n}$-algebra homomorphism $\theta: \mathbf{T}^{M} \rightarrow \mathbb{I}$ where $\mathbb{I}$ is an integrally closed finite torsion-free extension of $\Lambda_{n}$.

By interpolating the pseudo-representations associated to the arithmetic specializations of $\theta$ (see Sect.4.2), one constructs a continuous homomorphism $\rho_{\theta}: G_{\mathbb{Q}} \rightarrow \widehat{G}(\mathbb{I})$ associated to $\theta$ (see Lemma 4.3 in the text). We assume throughout that the residual representation is irreducible. Following the ideas of [H13b], we prove first, under some assumptions (mostly $\mathbb{I}=\Lambda_{n}$ and $\mathbb{Z}_{p}$-regularity), that if the Hida family $\theta$ is "generic", the image of $\rho_{\theta}$ is " $\Lambda_{n}$-full" (see 4.8); more precisely, there is a non-zero ideal $\mathfrak{l}$ of $\Lambda_{n}$ such that the image of Galois contains the principal congruence subgroup $\Gamma_{\widehat{G}^{\prime}}(\mathfrak{l})$ in $\widehat{G}^{\prime}\left(\Lambda_{n}\right)$, where $\widehat{G}^{\prime}$ denotes the derived group of $\widehat{G}$. An important point of the proof is to create a $\Lambda_{n}$-structure on the submodule of a Lie algebra associated to $\operatorname{Im} \rho_{\theta}$; in the case $n=1$, Hida [H13b] used conjugation by certain elements of the inertia ; a suitable generalization using repeated use of Poisson brackets provides the desired structure of $\Lambda_{n}$-module. Moreover, we ask the relation between the maximal such ideal $\mathfrak{l}_{\theta}$, called the Galois level of $\theta$, and the schematic intersection of the irreducible component $\operatorname{Spec}(\mathbb{I})$ with other components, more precisely, at least if $\mathbb{I}=\Lambda_{n}$, we ask whether the set of primes containing $\mathfrak{l}_{\theta}$ coincides with the union of the zero loci in $\Lambda_{n}$ of the greatest common divisor of the congruence ideal of $\theta$ and those of other non-generic families congruent to $\theta$. The equality of these has been established for $n=1$ by Hida [H13b]. We prove this equality for $n=2$ in the case of twisted Yoshida lift congruence. In a subsequent paper, we plan to formulate more precisely this relation (including exponents of height one prime ideals) and generalize it to more general groups. The question of replacing $\Lambda_{n}$-fullness by $\mathbb{I}$-fullness, or rather $\mathbb{I}^{\prime}$-fullness for a suitable subring of $\mathbb{I}$ is currently investigated, by a student of one of the authors ${ }^{1}$.

\section{Galois Representations associated to Siegel modular forms}

Let $G=\mathrm{GSp}_{2 n}$ be the Chevalley group of symplectic similitude matrices for $J$. Let $\nu: G \rightarrow \mathbb{G}_{m}$ be the similitude factor character. Its kernel $\mathrm{Sp}_{2 n}$ coincides with the derived group $G^{\prime}$ of $G$. We denote by $B=T U$ its standard Borel (consisting of upper triangular matrices in $G$ ).

Let $S_{k}^{(n)}(M)$ be the space of genus $n$ holomorphic Siegel cusp forms of weight $k=\left(k_{1}, \ldots, k_{n}\right)$ with $k_{1} \geq k_{2} \geq \ldots \geq k_{n} \geq n+1$, and level $M$ (say, for the principal congruence subgroup of level $M$ ). Let $\mathbf{T}_{k}^{M}$ be the $M$-spherical $\mathbb{Z}\left[\frac{1}{M}\right]$-algebra generated by the Hecke operators $T_{\ell, i}$ for all rational primes $\ell$ prime to $M$ and all $i=1, \ldots, n$, acting on $S_{k}^{(n)}(M)$. Recall that $T_{\ell, i}$ denotes the action of the double class $\Gamma(M) \cdot d_{\ell, i} \cdot \Gamma(M)$ where $d_{\ell, 1}=\operatorname{diag}\left(1_{n}, \ell \cdot 1_{n}\right)$ and $d_{\ell, i}=\operatorname{diag}\left(1_{n+1-i}, \ell \cdot 1_{2 i}, \ell^{2} \cdot 1_{n+1-i}\right)$ for $i=2, \ldots n$. Let $f \in S_{k}^{(n)}(M)$ be an eigenform for $\mathbf{T}_{k}^{M}$; let $K_{0}$ be a number field containing its eigenvalues; let $\mathcal{O}_{0}$ be its ring of integers. It gives rise to a character $\theta_{f}: \mathbf{T}_{k}^{M} \rightarrow \mathcal{O}_{0}$. For any prime $\ell$ not dividing $M$, we denote by $P_{f, \ell}(X)$ the Hecke polynomial (see [FC90] Chapt.VII, Sect.1); it is monic of degree $2^{n}$.

Fix an odd prime $p$ relatively prime to $M$, an embedding $\iota_{p}: \overline{\mathbb{Q}} \hookrightarrow \overline{\mathbb{Q}}_{p}$ and assume that $f$ is ordinary for $\iota_{p}$. It means that the multiset of $p$-adic valuations of the (images by $\iota_{p}$ of the) roots of the Hecke polynomial $P_{f, p}(X)$ coincides with the multiset $\left\{\sum_{i \in I}\left(k_{i}-i\right) ; I \subset[1, n]\right\}$ of Hodge weights.

To the data $(G, B, T)$, one can associate a dual reductive group over $\mathbb{Z}\left[\frac{1}{2}\right]$ with a standard Borel and a standard torus $(\widehat{G}, \widehat{B}, \widehat{T})$, with an identification $X^{*}(T)=X_{*}(\widehat{T})$. By comparing the root data (with épinglage, see [MT02] Sect.3.2.2), one has a canonical identification $\widehat{G}=\mathrm{GSpin}_{2 n+1}$ over $\mathbb{Z}\left[\frac{1}{2}\right]$. Therefore $\widehat{G}$ is endowed with a linear representation spin: $\widehat{G} \rightarrow \mathrm{GL}_{2^{n}}$ and an orthogonal representation $\pi: \widehat{G} \rightarrow \mathrm{SO}_{2 n+1}$ whose kernel is the center $\mathbb{G}_{m}$ of $\widehat{G}$ (the central inclusion $\mathbb{G}_{m} \rightarrow \widehat{G}$ is dual to the similitude factor $G \rightarrow \mathbb{G}_{m}$ ). It also carries a similitude factor $\widehat{G} \rightarrow \mathbb{G}_{m}$, dual to the central inclusion $\mathbb{G}_{m} \rightarrow G$; its kernel coincides with the derived group $\widehat{G}^{\prime}=\operatorname{Spin}_{2 n+1}$.

For any number field $F$, we put $G_{F}=\operatorname{Gal}(\bar{F} / F)$ and for any finite place $v$ of $F$, we denote by $D_{v}$ a decomposition subgroup at $v$ in $G_{F}$; let $\epsilon: G_{F} \rightarrow \mathbb{Z}_{p}^{\times}$be the $p$-adic cyclotomic character. One still denotes by $\epsilon$ its restriction to $D_{v}$. We assume that there exists a Galois representation $\rho_{f}: G_{\mathbb{Q}} \rightarrow \widehat{G}\left(\overline{\mathbb{Q}}_{p}\right)$ unramified outside $M p$ and such that for any rational prime $\ell$ not dividing $M p$, the

\footnotetext{
${ }^{1}$ H. Hida
} 
characteristic polynomial Char $\left(\operatorname{spin} \circ \rho_{f}\left(\operatorname{Frob}_{\ell}\right)\right)$ coincides with $\iota_{p}\left(P_{f, \ell}(X)\right)$. It is called the Galois representation associated to $f$. The integer $w=k_{1}+\cdots+k_{n}-\frac{n(n+1)}{2}$ is called the motivic weight of $\rho_{f}$.

The existence of $\rho_{f}$ is known for $n=1$ (Deligne) and for $n=2$ (due to R. Taylor, Laumon and Weissauer, in [Ta93], [We05] and [Lau05]). By compactness, there exists a $p$-adic field $K \subset \overline{\mathbb{Q}}_{p}$ such that $\rho_{f}$ is defined over $K$. We may assume that $\iota_{p}\left(K_{0}\right) \subset K$, hence if $\mathcal{O}$ denotes its valuation ring, we have $\iota_{p}\left(\mathcal{O}_{0}\right) \subset \mathcal{O}$. One can choose an $\mathcal{O}$-lattice stable in the orthogonal representation $\pi \circ \rho_{f}$. We assume it is unimodular. It implies that $\rho_{f}$ takes values in $\widehat{G}(\mathcal{O})$. Let $\varpi$ be a uniformizing parameter of $\mathcal{O}$ and $\mathbb{F}=\mathcal{O} / \varpi \mathcal{O}$ its residue field. Let $\bar{\rho}_{f}: G_{\mathbb{Q}} \rightarrow \widehat{G}(\mathbb{F})$ be the residual representation.

Under the assumption of "automorphic ordinarity", it is conjectured that the following "Galois ordinarity" holds. Let $\widetilde{\rho}=\sum_{i=1}^{n} \varpi_{i}$ be the character of $T$ sum of all the fundamental weights of $G$; it is given by $\rho$ on $T^{\prime}=T \cap G^{\prime}$ and by $z \mapsto z^{\frac{n(n+1)}{2}}$ on the center. Let us view $\lambda+\widetilde{\rho}$ as a cocharacter of $\widehat{T}$ via $X^{*}(T)=X_{*}(\widehat{T})$. For $\widehat{g}, \widehat{h} \in \widehat{G}$, let us put $\widehat{h}^{\widehat{g}}=\widehat{g}^{-1} \widehat{h} \widehat{g}$. Write $D_{p}$ for a (chosen) decomposition group at $p$ inside $\operatorname{Gal}(\overline{\mathbb{Q}} / \mathbb{Q})$ with the inertia subgroup $I_{p}$.

(GO) There exists $\widehat{g} \in \widehat{G}(\mathcal{O})$ such that $\rho_{f}\left(D_{p}\right) \subset \widehat{g} \cdot \widehat{B}(\mathcal{O}) \cdot \widehat{g}^{-1}$ and for any $\sigma \in I_{p}, \rho_{f}^{\widehat{g}}(\sigma)(\bmod \widehat{N}(\mathcal{O}))$ is given by $(\lambda+\widetilde{\rho}) \circ \epsilon(\sigma)$.

"Automorphic ordinarity" implies "Galois ordinarity" is known for $n=1$ (due to Wiles) and for $n=2$ (due to Urban [Ur05]). We assume in the sequel it holds for any $n$. It would follow from Katz-Messing theorem if one knew that spin $\circ \rho_{f}$ is motivic.

In order to motivate the assumptions we shall make in genus $n$, let us prove some results in genus 2 , where the results have less conditions.

\section{Fullness of the image for Galois Representations in GSp(4)}

3.1. Irreducibility and open image. Let $n=2$ and $f$ be a genus 2 cusp eigenform of weight $k=\left(k_{1}, k_{2}\right), k_{1} \geq k_{2} \geq 3$, level $M$, as above. Fix an odd prime $p$ prime to $M$ at which $f$ is ordinary; let $P_{f, p}(X)=(X-\alpha)(X-\beta)(X-\gamma)(X-\delta)$ be the Hecke polynomial of $f$ at $p$, with roots ordered with increasing $p$-adic valuation $\left(\right.$ so that $\operatorname{ord}_{p}(\alpha)=0, \operatorname{ord}_{p}(\beta)=k_{2}-2, \operatorname{ord}_{p}(\gamma)=k_{1}-1$ and $\left.\operatorname{ord}_{p}(\alpha)=k_{1}+k_{2}-3\right)$; we assume that all the Hecke eigenvalues, together with $\alpha, \beta, \gamma$ and $\delta$ the are contained in $\mathcal{O}$ and that the Galois representation associated to $f$ ([Ta93], [We05] and [Lau05]) is defined over $\mathcal{O}: \rho_{f}: G_{\mathbb{Q}} \rightarrow \operatorname{GSp}_{4}(\mathcal{O})$ where the group of symplectic similitudes is relative to the matrix $J=\left(\begin{array}{cccc}0 & 0 & 0 & 1 \\ 0 & 0 & 1 & 0 \\ 0 & -1 & 0 & 0 \\ -1 & 0 & 0 & 0\end{array}\right)$. Recall that the spin representation provides an isomorphism $\mathrm{GSpin}_{5} \cong \mathrm{GSp}_{4} \subset \mathrm{GL}_{4}$. Let $(L, \psi)$ be the $\mathcal{O}$-module of the representation endowed with a unimodular symplectic pairing $\psi$; we fix a symplectic $\mathcal{O}$-basis $\left(e_{1}, e_{2}, e_{3}, e_{4}\right)$ of $L$ with $\psi\left(e_{1}, e_{4}\right)=\psi\left(e_{2}, e_{3}\right)=1$ so that we have an identification $\operatorname{GSp}(L, \psi)=\operatorname{GSp}_{4}(\mathcal{O})$; let $V=L\left[\frac{1}{p}\right]$ be the $K$-vector space spanned by $L$. We have

$$
\left.\rho_{f}\right|_{D_{p}} \sim\left(\begin{array}{cccc}
\epsilon^{k_{1}+k_{2}-3} u r\left(\frac{\delta}{p^{k_{1}+k_{2}-3}}\right) & * & * & * \\
0 & \epsilon^{k_{1}-1} u r\left(\frac{\gamma}{p^{k_{1}-1}}\right) & * & * \\
0 & 0 & \epsilon^{k_{2}-2} u r\left(\frac{\beta}{p^{k_{2}-2}}\right) & * \\
0 & 0 & 0 & \operatorname{ur}(\alpha)
\end{array}\right) .
$$

Here $\operatorname{ur}(x): D_{p} \rightarrow \mathcal{O}^{\times}$is the unramified character sending Frob $\operatorname{Fo}_{p}$ to $x \in \mathcal{O}^{\times}$. We shall assume in the sequel that the conjugation needed for this description is the identity. We also put $\bar{\rho}: G_{\mathbb{Q}} \rightarrow \operatorname{GSp}_{4}(\mathbb{F})$ its reduction modulo $\varpi$.

We first show

Proposition 3.1. If $f$ is neither CAP nor endoscopic, and if either

(i) Langlands transfer holds from $\mathrm{GSp}(4)$ to $\mathrm{GL}(4)$ (no need of the ordinarity assumption then),

(ii) $p>7$, the semisimplification $\left(\left.\bar{\rho}\right|_{G_{\mathbb{Q}(\zeta p)}}\right)^{\text {ss }}$ contains a copy of $\mathrm{SL}_{2}\left(\mathbb{F}_{p}\right)$, and the $p$-adic units $\frac{\delta}{p^{k_{1}+k_{2}-3}}, \frac{\gamma}{p^{k_{1}-1}}, \frac{\beta}{p^{k_{2}-2}}$ and $\alpha$ are distinct modulo $\varpi$,

then $\rho_{f}$ is absolutely irreducible. 
In the first case, one can use [CaGe13]. The transfer has been established by Arthur for $\operatorname{Sp}(4)$, but not yet for $\operatorname{GSp}(4)$, unless $f$ admits a generic form with the same eigenvalues.

In the second case, we proceed by case inspection. If there is a stable line $D=\left\langle e_{2}^{\prime}\right\rangle$ in $L$, let $P_{1}=\left\langle e_{2}^{\prime}, e_{3}^{\prime}\right\rangle$ be a hyperbolic plane of $L$ containing $D$ and $P_{2}=\left\langle e_{1}^{\prime}, e_{4}^{\prime}\right\rangle$ its orthogonal, so that $P_{1} \perp P_{2}=L$ be a decomposition of $L$ into two orthogonal hyperbolic planes (it is possible by unimodularity of the symplectic pairing on $L)$. By taking $\left(e_{1}^{\prime}, e_{2}^{\prime}, e_{3}^{\prime}, e_{4}^{\prime}\right)$ as symplectic basis of $L$ adapted to this decomposition, we see in this basis that

$$
\rho_{f}=\left(\begin{array}{cccc}
* & 0 & \bullet & * \\
\bullet & \chi & \bullet & \bullet \\
0 & 0 & \chi^{\prime} & 0 \\
* & 0 & \bullet & *
\end{array}\right) .
$$

hence,

- $\rho_{f}$ admits a degree 1 subrepresentation $\chi$ which is locally algebraic at $p$ and finitely ramified, hence it is given by an $\left(A_{0}\right)$-type Hecke character of $\mathbb{Q}$, which cannot be of finite order because $\chi(p)$ cannot be of archimedean absolute value one by Deligne purity for $\alpha, \beta, \gamma$ and $\delta$, so that $\chi$ cannot have Hodge-Tate weight 0 : it must be $k_{2}-2$ or $k_{1}-1$

- $\rho_{f}$ has a degree 2 subquotient $\sigma=\left(\begin{array}{cc}* & * \\ * & *\end{array}\right)$, which is odd because its determinant is the similitude factor of $\rho$, which is odd.

Moreover, $\sigma$ is $p$-ordinary with two distinct Hodge-Tate weights $\left(0\right.$ and $\left.k_{1}+k_{2}-3\right)$ and its residual representation is irreducible over $G_{\mathbb{Q}\left(\zeta_{p}\right)}$ by assumption. Therefore, the representation $\sigma$ is modular : $\sigma=\sigma_{g}$ for an ordinary form $g$ of weight $\ell \geq 2$ and level prime to $p$ by Emerton's theorem on FontaineMazur Conjecture ([Em14, Cor.1.2.2]) ; this implies by the classification of Vogan-Zuckerman (see [Ta93] Section 1 or [Ti09] Section 6) that, $k_{1}=k_{2}=k, \ell=2 k-2, \chi=\epsilon^{k-1} \cdot$ (finite), and that the $L$-function of $f$ is of the form $L(\chi, s) L(g, s) L(\chi, s-1)$, hence $f$ is CAP of Siegel type (i.e. is a Saito-Kurokawa lift), contrary to the assumption.

Similarly, if there is a stable isotropic plane, $\rho_{f}$ contains a two-dimensional subrepresentation; it is $p$-ordinary with distinct Hodge-Tate weights, $p>7$, and the image of its reduction modulo $\varpi$ restricted to $G_{\mathbb{Q}\left(\zeta_{p}\right)}$ contains $\mathrm{SL}_{2}(\mathbb{F})$, it is odd by Cor.1.3 of Calegari [Ca13] ; so again by [Em14, Cor.1.2.2], Fontaine-Mazur Conjecture holds and shows that $f$ is CAP of Klingen type, contradiction. Finally, if there is a stable hyperbolic plane, we see by a similar argument (but without using Calegari's argument, hence without assuming $p>7$ and the $\mathrm{SL}_{2}\left(\mathbb{F}_{p}\right)$ condition, because the oddness is obvious in this case), that $f$ is a Yoshida lift, so it is endoscopic. QED.

Proposition 3.2. Assume that the adjoint representation of $\rho_{f}$ on the Lie algebra $\mathfrak{s p}_{4}$ is irreducible. Then (without assuming ordinarity), the image of Galois is full, i.e. contains a conjugate in $G\left(\overline{\mathbb{Q}}_{p}\right)$ of a congruence subgroup of $G\left(\mathbb{Z}_{p}\right)$.

We first show that the image of Galois is open in its Zariski closure. Say that $\operatorname{Im} \rho_{f} \subset \operatorname{GSp}_{4}(E)$ for a finite extension $E / \mathbb{Q}_{p}$, and let $G=\operatorname{Res}_{E / \mathbb{Q}_{p}} \mathrm{GSp}_{4}$. Let $\mathfrak{g}$, resp. $\mathfrak{G}$, be the Lie algebra over $\mathbb{Q}_{p}$ of image of Galois (with its natural structure of $p$-adic Lie group), resp. of its $\mathbb{Q}_{p}$-Zariski closure in $G$. For any embedding $\sigma: E \rightarrow \overline{\mathbb{Q}}_{p}$, let $\mathfrak{G}_{\sigma}$ resp. $\mathfrak{g}_{\sigma}$ be the $\sigma$-projection of $\mathfrak{G} \otimes \overline{\mathbb{Q}}_{p}$ resp. $\mathfrak{g} \otimes \overline{\mathbb{Q}}_{p} ;$ By a theorem of Chevalley [Che51, p.177, Th.15], we see that, for any $\sigma$, the derived Lie algebras $\mathfrak{g}_{\sigma}^{\prime}$ and $\mathfrak{G}_{\sigma}^{\prime}$ coincide. By absolute irreducibility of $\rho_{f}$, the center of $\mathfrak{g}_{\sigma}$ has rank at most one. Moreover, since the similitude factor $\nu \circ \rho$ is a non-trivial power of the $p$-adic cyclotomic character, the rank of the center is exactly one for any $\sigma$, so that $\mathfrak{g} \otimes \overline{\mathbb{Q}}_{p}=\mathfrak{G} \otimes \overline{\mathbb{Q}}_{p}$.

The possibilities for $\mathfrak{G}^{\prime} \otimes \overline{\mathbb{Q}}_{p}$ are listed below

(F) $\mathfrak{G}^{\prime} \otimes \overline{\mathbb{Q}}_{p} \sim \mathfrak{s p}_{4}$.

(Y) $\mathfrak{G}^{\prime} \otimes \overline{\mathbb{Q}}_{p} \sim \mathfrak{s l}(2) \times \mathfrak{s l}(2)$

$(\mathrm{K}) \mathfrak{G}^{\prime} \otimes \overline{\mathbb{Q}}_{p} \sim \mathfrak{s l}(2)$ with $\mathfrak{s l}(2)$ embedded into a Klingen parabolic subalgebra.

(C) $\mathfrak{G}^{\prime} \otimes \overline{\mathbb{Q}}_{p} \sim \mathfrak{s l}(2)$ via the symmetric cube representation of $\mathrm{SL}(2)$,

(S) $\mathfrak{G}^{\prime} \otimes \overline{\mathbb{Q}}_{p} \sim \mathfrak{s l}(2)$ with $\mathfrak{s l}(2)$ embedded into a Siegel parabolic subalgebra.

(T) $\mathfrak{G}^{\prime} \otimes \overline{\mathbb{Q}}_{p} \sim\{1\}$ 
Let us show that in all cases except $(F)$, the adjoint representation of $\rho_{f}$ on $\mathfrak{g s p}_{4}$ (denoted by adding the superscript "ad") would be reducible. Indeed, in all cases except $(F)$, we see that $\mathfrak{G}^{\text {ad }}$ is reducible (note that $\left(\mathrm{Symm}^{3} \mathfrak{s l}(2)\right)^{\text {ad }}$ is also reducible). This shows that the $\mathbb{Q}_{p}$-Lie algebra $\mathfrak{g}$ of $\operatorname{Im} \rho_{f}$ is a $\mathbb{Q}_{p}$-form of $\mathfrak{g s p}_{4}\left(\mathbb{Q}_{p}\right)$ contained in $\mathfrak{g s p}_{4}\left(\overline{\mathbb{Q}}_{p}\right)$. Since $\operatorname{Aut}\left(\mathfrak{g s p}_{4}\right)=\operatorname{Int}\left(\mathfrak{g s p}_{4}\right)$, it implies that there exists $\alpha \in G\left(\overline{\mathbb{Q}}_{p}\right)$ such that

$$
\mathfrak{g}=\alpha \cdot \mathfrak{g s p}_{4}\left(\mathbb{Q}_{p}\right) \cdot \alpha^{-1} .
$$

In order to get a more complete result without assuming adjoint irreducibility, one needs to analyze the connected components of the Galois image.

3.2. Connected components of the image of Galois. Let $\rho=\left\{\rho_{l}\right\}_{l}$ be an $n$-dimensional compatible system of $l$-adic representations of $\mathrm{Gal}(\overline{\mathbb{Q}} / k)$ satisfying the assertions of [P98a, Theorems 3.23]. Write $V_{l} \cong \mathbb{Q}_{l}^{n}$ for the space of $\rho_{l}$. Replacing $\rho_{l}$ by its semi-simplification, we may assume that $\rho_{l}$ is semi-simple for all $l$. Define an algebraic group $G_{p / \mathbb{Q}_{p}}$ over $\mathbb{Q}_{p}$ by the $\mathbb{Q}_{p}$-Zariski closure in $\operatorname{GL}\left(V_{p / \mathbb{Q}_{p}}\right)$ of $\operatorname{Im}\left(\rho_{p}\right)$ for a prime $p$, and let $G_{p}^{\circ}$ be its connected component. Since $\rho_{p}$ is semi-simple, $G_{p}$ is reductive. Then by a result of Serre, $G_{p} / G_{p}^{\circ}$ and $\operatorname{rank} G_{p}^{\circ}$ are independent of $p$ (see [P98a, Theorem 3.6]). Let $k^{\text {conn }}$ for the fixed field of $\rho_{p}^{-1}\left(G_{p}^{\circ}\left(\mathbb{Q}_{p}\right)\right)$. We fix a prime $p$ and write $\mathfrak{l}$ for a prime ideal of $k^{\text {conn }}$ prime to $p$. Let $\rho_{p}\left(\text { Frob }_{\mathfrak{l}}\right)^{s s}$ be the semi-simplification of $\rho_{p}\left(\right.$ Frob $\left._{\mathfrak{l}}\right)$ inside $G_{p}\left(\mathbb{Q}_{p}\right)$. Write $T_{\mathfrak{l}}$ for the smallest algebraic subgroup over $\mathbb{Q}_{p}$ containing $\rho(\text { Frobl })^{s s}$ inside $G_{p}^{\circ}\left(\mathbb{Q}_{p}\right)$. Then the connected component $T_{\mathfrak{l}}^{\circ}$ is a torus, and it is isomorphic to a base-change to $\mathbb{Q}_{p}$ of a $\mathbb{Q}$-torus in $\mathrm{GL}(n)$. If $T_{\mathfrak{l}}$ is a maximal torus of $G_{p}^{\circ}$, then $\rho_{p}\left(\mathrm{Frob}_{\mathfrak{l}}\right)$ is already semi-simple.

Lemma 3.3 (Serre). Let $p$ be a prime. The algebraic group $G_{p}^{\circ}$ has a dense Zariski open subset $U$ stable under conjugation such that $T_{l}$ is connected and maximal if $\rho_{p}\left(\mathrm{Frob}_{l}\right)^{s s}$ is in $U\left(\mathbb{Q}_{p}\right)$. In particular, for any prime $\mathfrak{l}$ in a density one subset of primes of $k^{\text {conn }}, T_{\mathfrak{l}}$ is maximal.

This follows from the argument in [Ch92, Theorem 3.7]. Though Chi assumes that $\rho$ comes from an abelian variety, his argument works in general.

3.3. Connected semi-simple subgroups of $\operatorname{GSp}(4)$. Let $\rho=\left\{\rho_{\mathfrak{l}}\right\}_{\mathfrak{r}}$ be a strict compatible system of symplectic semi-simple representations of $\operatorname{Gal}(\bar{k} / k)$ into $\operatorname{GSp}_{4}\left(T_{\mathfrak{l}}\right)$ for a number field $T$ (associated to a motive). Assume that $T$ is the smallest coefficient field (i.e., it is generated by trace of Frobenii). Here $\mathfrak{l}$ runs over primes of $T$. Let $l$ be the residual characteristic of $\mathfrak{l}$. Let $\operatorname{Res} \rho=\left\{\rho_{l}\right\}_{l}$ for $\rho_{l}=\prod_{\mathfrak{r} \mid l} \rho_{\mathfrak{l}}$ regarded to have values in $\operatorname{GSp}_{4}\left(T \otimes_{\mathbb{Q}} \mathbb{Q}_{l}\right)$. Then $\operatorname{Res} \rho$ is a compatible system with coefficients in $\mathbb{Q}$ acting on the $\mathbb{Q}_{l}$-vector spaces $V_{l}=\left(T \otimes_{\mathbb{Q}} \mathbb{Q}_{l}\right)^{4}$. Consider the $\mathbb{Q}_{l}$-Zariski closure $G_{l}$ of $\operatorname{Im}\left(\rho_{l}\right)$ in $\mathrm{GL}\left(V_{l}\right)$.

As mentioned earlier, $G_{l}$ is a reductive subgroup of $\mathrm{GL}\left(V_{l}\right)$ defined over $\mathbb{Q}_{l}, \operatorname{rank} G_{l}^{\circ}$ is independent of $l$ and $G_{l} / G_{l}^{\circ}$ is independent of $l$ and is isomorphic to $\operatorname{Gal}\left(k^{c o n n} / k\right)$ for the fixed field $k^{\text {conn }}$ of $\rho_{l}^{-1}\left(G_{l}^{\circ}\right)$

Since $G_{l}$ commutes with the action of $T$ on $V_{l}$, for each prime factor ll, we have its projection $G_{\mathfrak{l}}$ in $\operatorname{GSp}_{4}\left(T_{\mathfrak{l}}\right)$. Let $G_{l}^{\circ}$ be the connected component of $G_{l}$ (and $G_{\mathfrak{l}}^{\circ}$ for the l-component of $G_{l}^{\circ}$; so, $G_{\mathfrak{l}}^{\circ}$ is the connected component of $G_{\mathfrak{l}}$ ).

Let $G_{\mathfrak{l}}^{\prime}$ be the derived group of $G_{\mathfrak{l}}^{\circ}$. We may regard $G_{\mathfrak{l}}^{\prime} \subset \operatorname{Res}_{T_{\mathfrak{l}} / \mathbb{Q}_{l}} \operatorname{Sp}(4)$. Thus the projections of $G_{\mathfrak{l}}^{\prime}\left(\overline{\mathbb{Q}}_{l}\right)$ to each simple component $\operatorname{Sp}_{4}\left(\overline{\mathbb{Q}}_{l}\right)$ of $\left(\operatorname{Res}_{T_{l} / \mathbb{Q}_{l}} \operatorname{Sp}(4)\right)\left(\overline{\mathbb{Q}}_{l}\right)$ are mutually isomorphic. We write $G_{\mathfrak{l}}^{\prime} \sim G^{\prime}$ for a semi-simple connected subgroup $G^{\prime}$ of $\operatorname{Sp}(4) \overline{\mathbb{Q}}_{l}$ if its projections to any simple factor $\operatorname{Sp}_{4}\left(\overline{\mathbb{Q}}_{l}\right)$ of $\operatorname{Res}_{T_{l} / \mathbb{Q}_{l}} \operatorname{Sp}(4) / \overline{\mathbb{Q}}_{l}$ are isomorphic to $G^{\prime}$ over $\overline{\mathbb{Q}}_{l}$. Assume that $\rho$ has weight $w$, i.e., $\operatorname{det} \rho$ is, up to finite character, given by $\left\{\epsilon_{l}^{w}\right\}_{l}$ for the $l$-adic cyclotomic character $\epsilon_{l}$.

Let $\mathcal{G}$ be a connected semi-simple subgroup of $\operatorname{Sp}(4)_{/ k}$ for a field $k$, and write $\mathfrak{g}$ for its Lie algebra. Write $V_{n}$ for the symplectic space of dimension $2 n$ on which $\operatorname{Sp}(2 n)$ acts. Then by [LS98, Theorem 1], the largest proper semi-simple connected subgroup $\mathcal{G}$ of $\mathrm{Sp}(4)$ is isomorphic to $\mathrm{SL}(2) \times \mathrm{SL}(2)$ over an algebraic closure $\bar{k}$. Making an identification $V_{2} \cong V_{1} \oplus V_{1}$ as symplectic spaces, this group $\mathcal{G}$ acts diagonally on $V_{1} \oplus V_{1}$. As for smaller semi-simple connected subgroups, we have two possibilities $\mathcal{G}=1$ and $\mathcal{G} \cong \mathrm{SL}(2)$. The second possibility includes the case where the isomorphism $\mathrm{SL}(2) \cong \mathcal{G}$ is given by the symmetric cube representation of $\mathrm{SL}(2)$. Thus irreducibility of $\mathcal{G}$-module $\mathfrak{g}$ (under the 
adjoint action) is satisfied if the derived group $\mathcal{G}$ is either $\mathrm{SL}(2)$ (the image of symmetric cube) or $\operatorname{Sp}(4)$.

For a prime $\mathfrak{P}$ over $p$ of $k$, we suppose to have

$$
\left.\rho\right|_{I_{\mathfrak{P}}} \sim\left(\begin{array}{cccc}
\epsilon^{w_{1}} & * & * & * \\
0 & \epsilon^{w_{2}} & * & * \\
0 & 0 & \epsilon^{w_{3}} & * \\
0 & 0 & 0 & \epsilon^{w_{4}}
\end{array}\right) \quad\left(w_{1}>w_{2}>w_{3}>w_{4}\right)
$$

up to finite error for the $p$-adic cyclotomic character $\epsilon=\epsilon_{p}$ of the inertia group $I_{\mathfrak{P}}$ of $\mathfrak{P}$. Thus we have six possibilities of the derived group $G_{\mathfrak{p}}^{\prime}$ (the group $G_{\mathfrak{p}}^{\circ}$ is split over $T_{\mathfrak{p}}$ by (Reg)):

(F) $G_{\mathfrak{p}}^{\prime} \sim \operatorname{Sp}(4)$.

(Y) $G_{\mathfrak{p}}^{\prime} \sim \mathrm{SL}(2) \times \mathrm{SL}(2)$ with $\rho_{\mathfrak{p}}^{\text {conn }}=\rho_{1, \mathfrak{p}} \oplus \rho_{2, \mathfrak{p}}$, where $\rho_{1, \mathfrak{p}}$ is not twist equivalent to $\rho_{2, \mathfrak{p}}$.

(K) $G_{\mathfrak{p}}^{\prime} \sim \mathrm{SL}(2)$ with $\rho_{\mathfrak{p}}^{\text {conn }}=\chi \oplus \chi^{\prime} \oplus \rho_{1, \mathfrak{p}}$ with irreducible two dimensional $\rho_{1, \mathfrak{p}}$.

(C) $G_{\mathfrak{p}}^{\prime} \sim \mathrm{SL}(2)$ via the symmetric cube representation of $\mathrm{SL}(2)$.

(S) $G_{\mathfrak{p}}^{\prime} \sim \mathrm{SL}(2)$ with $\rho_{\mathfrak{p}}^{\text {conn }}=\rho_{1, \mathfrak{p}} \oplus\left(\rho_{1, \mathfrak{p}} \otimes \chi\right)$ with absolutely irreducible two dimensional $\rho_{1, \mathfrak{p}}$.

(T) $G_{\mathfrak{p}}^{\prime} \sim\{1\}$ with $\rho_{\mathfrak{p}}=\operatorname{Ind}_{F}^{\mathbb{Q}} \chi$ for a degree $4 \mathrm{CM}$ field $F / \mathbb{Q}$.

Here we write $\rho_{\mathfrak{p}}^{\text {conn }}$ for the restriction of $\rho_{\mathfrak{p}}$ to $\operatorname{Gal}\left(\overline{\mathbb{Q}} / k^{\text {conn }}\right)$.

3.4. $\mathbb{Z}_{p}$-Fullness for $\operatorname{GSp}(4)$ and its subgroups. Note that $\operatorname{Sp}_{4} \cong \operatorname{Spin}_{5}$ over $\mathbb{Z}_{p}$ (for $p>2$ ). Suppose that $\rho$ is the (semi-simple) system associated to a Siegel cusp form (so $k=\mathbb{Q}$ ) with regular weight and that $\rho_{\mathfrak{p}}$ (for $\mathfrak{p} \mid p$ ) is ordinary as in Proposition 3.1. Recall that $G_{\mathfrak{p}}$ is the Zariski closure of $\operatorname{Im}\left(\rho_{\mathfrak{p}}\right)$, that $G_{\mathfrak{p}}^{\circ}$ is the identity connected component of $G_{\mathfrak{p}}$ and that $G_{\mathfrak{p}}^{\prime}$ is the derived group of $G_{\mathfrak{p}}^{\circ}$ for a prime $\mathfrak{p} \mid p$ of $T$. For a semi-simple $\mathbb{Q}$-split subgroup $G$ of $\operatorname{Sp}(4)$ and a semi-simple $T_{\mathfrak{p}}$ subgroup $H$, we write $H \sim G$ if $H \cong G \times_{\mathbb{Q}} T_{\mathfrak{p}}$. Then as seen in Section 3.3, we have the following six possibilities of $G_{\mathfrak{p}}^{\prime}$ :

(F) $G_{\mathfrak{p}}^{\prime} \sim G$ for $G=\operatorname{Sp}(4)$.

(Y) $G_{\mathfrak{p}}^{\prime} \sim G$ for $G=\mathrm{SL}(2) \times \mathrm{SL}(2)$ with $\rho_{\mathfrak{p}}^{\text {conn }}=\rho_{1, \mathfrak{p}} \oplus \rho_{2, \mathfrak{p}}$, where $\rho_{1, \mathfrak{p}}$ is not twist equivalent to $\rho_{2, \mathfrak{p}}$.

(K) $G_{\mathfrak{p}}^{\prime} \sim G$ for $G=\mathrm{SL}(2)$ with $\rho_{\mathfrak{p}}^{\text {conn }}=\chi \oplus \chi^{\prime} \oplus \rho_{1, \mathfrak{p}}$ with irreducible two dimensional $\rho_{1, \mathfrak{p}}$.

(C) $G_{\mathfrak{p}}^{\prime} \sim G$ for $G=\mathrm{SL}(2)$ via the symmetric cube representation of $\mathrm{SL}(2)$.

(S) $G_{\mathfrak{p}}^{\prime} \sim G$ for $G=\mathrm{SL}(2)$ with $\rho_{\mathfrak{p}}^{\text {conn }}=\rho_{1, \mathfrak{p}} \oplus\left(\rho_{1, \mathfrak{p}} \otimes \chi\right)$ with absolutely irreducible two dimensional $\rho_{1, \mathfrak{p}}$.

(T) $G_{\mathfrak{p}}^{\prime} \sim\{1\}$ with $\rho_{\mathfrak{p}}=\operatorname{Ind}_{F}^{\mathbb{Q}} \chi$ for a degree $4 \mathrm{CM}$ field $F / \mathbb{Q}$.

Here $\operatorname{Im}\left(\rho_{j, \mathfrak{p}}\right)(j=1,2)$ contains an open subgroup of $\mathrm{SL}_{2}\left(\mathbb{Z}_{p}\right)$ by the definition of $G_{\mathfrak{p}}^{\prime}$, and $\chi, \chi^{\prime}$ are characters. In the automorphic side, Case $(\mathrm{Y})$ corresponds to the Yoshida lift from an automorphic form $\mathrm{GL}(2) \times \mathrm{GL}(2) / \mathbb{Q}$ or $\operatorname{Res}_{F / \mathbb{Q}} \mathrm{GL}(2)$ for a real quadratic field $F$, under the hypothesis that the starting automorphic form is not endo-scopic (i.e., non-CM). Cases (K) and (S) are either $\mathrm{CAP}$ or Eisenstein associated to the Siegel parabolic (S) and Klingen parabolic (K), assuming the corresponding $\mathrm{GL}(2)$ automorphic form is not endoscopic. Case $(\mathrm{C})$ is the symmetric cube lift from $\mathrm{GL}(2) / \mathbb{Q}$.

If $G$ is absolutely simple, let $\mathfrak{g}$ be the Lie algebra of $G$ and put $\mathfrak{g}_{\mathfrak{p}}=\mathfrak{g} \otimes_{\mathbb{Q}} T_{\mathfrak{p}}$ and regard it as the Lie algebra of $G_{\mathfrak{p}}^{\prime}$. Since $\operatorname{Im}\left(\rho_{\mathfrak{p}}\right)$ is Zariski dense in $G_{\mathfrak{p}}^{\prime}$, the adjoint action of $\rho_{\mathfrak{p}}$ is absolutely irreducible. Write $\operatorname{Ad}\left(\rho_{\mathfrak{p}}\right)$ for the adjoint representation acting on $\mathfrak{g}_{\mathfrak{p}}$, and let $E_{\mathfrak{p}}$ be the subfield of $T_{\mathfrak{p}}$ generated over $\mathbb{Q}_{p}$ by $\operatorname{Tr}\left(A d\left(\rho_{\mathfrak{p}}\right)\right)$ on the Galois group. Let $W$ be the p-adic integer ring of $E_{\mathfrak{p}}$. If $G=\mathrm{SL}(2) \times \mathrm{SL}(2)$ (i.e., we are in Case $(\mathrm{Y}))$, we consider $A d\left(\rho_{j, \mathfrak{p}}\right)$ acting on $\mathfrak{s l}_{2}\left(T_{\mathfrak{p}}\right)$. We then define $E_{j}$ by the subfield of $T_{\mathfrak{p}}$ generated over $\mathbb{Q}_{p}$ by the values of $\operatorname{Tr}\left(\operatorname{Ad}\left(\rho_{j, \mathfrak{p}}\right)\right)$ over $\operatorname{Gal}\left(\overline{\mathbb{Q}} / k^{\text {conn }}\right)$. Then we write $W_{j}$ for the $\mathfrak{p}$-adic integer ring of $E_{j}$.

Proposition 3.4. Let the notation be as above. Suppose $p>3$. If $G$ is absolutely simple, $\operatorname{Im}\left(\rho_{\mathfrak{p}}\right)$ contains an open subgroup of $G(W)$. If $G=\mathrm{SL}(2) \times \mathrm{SL}(2)$ (i.e., in Case $(\mathrm{Y}))$, then $\operatorname{Im}\left(\rho_{\mathfrak{p}}\right)$ contains an open subgroup of $\mathrm{SL}_{2}\left(W_{1}\right) \times \mathrm{SL}_{2}\left(W_{2}\right)$.

Proof. Suppose first that $G$ is absolutely simple (i.e., we are not in Case (Y)); so, $G$ is either $\mathrm{SL}(2)$ or $\mathrm{Sp}(4)$. Thus the adjoint representation of $G$ on its Lie algebra $\mathfrak{g}$ is absolutely irreducible. We 
regard $A d\left(\rho_{\mathfrak{p}}\right)$ restricted to $\mathrm{Gal}\left(\overline{\mathbb{Q}} / k^{\text {conn }}\right)$ as having values in $G^{\text {ad }}$ (the adjoint group of $G$ ). Since $\operatorname{Im}\left(\rho_{\mathfrak{p}}^{\text {conn }}\right)$ is Zariski dense in $G, \operatorname{Ad}\left(\rho_{\mathfrak{p}}^{\text {conn }}\right)$ is absolutely irreducible. Then by a theorem of Weisfeiler (see [P98b, Theorem 0.7]), there exists a linear algebraic group $H$ defined over $E_{\mathfrak{p}}$ in $T_{\mathfrak{p}}$ such that $H \times_{E_{\mathfrak{p}}} T_{\mathfrak{p}}=G^{a d} \times_{\mathbb{Q}} T_{\mathfrak{p}}$ with $\operatorname{Im}\left(\operatorname{Ad}\left(\rho_{\mathfrak{p}}^{\text {conn }}\right)\right.$ being an open subgroup of $H(W)$. Since the Siegel cusp form has regular weight, $\operatorname{Im}\left(\rho_{\mathfrak{p}}^{\text {conn }}\right)$ contains $p$-inertia regular element $g$ such that any of its power $g^{n}(0<n \in \mathbb{Z})$ has a split torus of maximal rank in $G$ as its centralizer. Thus $H$ is split, and the result follows as $G \rightarrow G^{a d}$ is a central isogeny.

Now we assume that we are in Case (Y). Applying the above argument to $\rho_{j, \mathfrak{p}}, \operatorname{Im}\left(\rho_{j, \mathfrak{p}}\right)$ contains an open subgroup of $\mathrm{SL}_{2}\left(W_{j}\right)$. Thus we need to show that $\operatorname{Ad}\left(\rho_{1}\right) \times \operatorname{Ad}\left(\rho_{2}\right)$ contains an open subgroup of $\mathrm{PGL}_{2}\left(W_{1}\right) \times \mathrm{PGL}_{2}\left(W_{2}\right)$. Let $P G$ be PGL(2) defined over $F$; so, $P G(F)=\mathrm{PGL}_{2}\left(T_{\mathfrak{p}}\right) \times \mathrm{PGL}_{2}\left(T_{\mathfrak{p}}\right)$. By [P98b, Main Theorem 0.2 (a)], there exist a semi-simple $\mathbb{Q}_{p}$-subalgebra $E$ of $F:=T_{\mathfrak{p}} \oplus T_{\mathfrak{p}}$ and a connected adjoint group $H_{/ E}$ and an isogeny $\varphi: H \times_{E} F \rightarrow P G$ such that $\left.\operatorname{Im}\left(\rho_{\mathfrak{p}}^{\text {conn }}\right)\right)$ is an open subgroup of $\varphi(H(E))$. If $E$ is a field, we have $E=E_{1}=E_{2}$, and $\rho_{1, \mathfrak{P}} \cong \rho_{2, \mathfrak{p}}$, and hence we are in Case (S), a contradiction. Thus $E=E_{1} \oplus E_{2}$, and $H(E)=\mathrm{PGL}_{2}\left(E_{1}\right) \times \mathrm{PGL}_{2}\left(E_{2}\right)$ as desired.

3.5. Pink's Lie algebra theory. Let $G$ be a split (smooth) connected reductive $\mathbb{Z}_{p}$-group with maximal split torus $T$. Let $G^{\prime}$ be the derived group of $G$, and put $\Gamma_{A}^{G}(\mathfrak{c})$ to be the kernel of the reduction map $G^{\prime}(A) \rightarrow G^{\prime}(A / \mathfrak{c})$ for a non-zero ideal $\mathfrak{c}$ of $A$ for a $\mathbb{Z}_{p^{-}}$algebra $A$. For a $\mathbb{Z}_{p^{-}}$ algebra $B \subset A$, a subgroup $H$ of $G(A)$ is $B$-full if $H \supset \Gamma_{B}^{G}(\mathfrak{c})$ for a non-zero $B$-ideal $\mathfrak{c}$. Let $\rho: \operatorname{Gal}(\overline{\mathbb{Q}} / K) \rightarrow G(A)$ be a continuous representation for a finite extension $K / \mathbb{Q}$. Suppose $A$ is an integral domain with quotient field $Q(A)$. We call $\rho B$-full with respect to $G$ if after replacing $\rho$ by a conjugate of $\rho$ under an element of $G(Q(A)), \operatorname{Im}(\rho)$ is $B$-full. Suppose that $B$ is a local ring with maximal ideal $\mathfrak{m}_{B}$. We call $\rho$ B-regular if $\operatorname{Im}(\rho)$ contains a regular element $b$ in the torus $T(B)$ such that $\alpha(b) \not \equiv \beta(b) \bmod \mathfrak{m}_{B}$ for all distinct roots $\alpha$ and $\beta$.

Via Pink's theory of tight correspondence between $p$-profinite Lie subalgebras of $\mathfrak{s l}(2)$ and $p$ profinite subgroups, we want to show that if a $p$-profinite subgroup of $\operatorname{Spin}_{2 n+1}$ contains sufficiently large unipotent subgroups, it is full. Let us recall Pink's theory briefly. To study a general $p$ profinite subgroup $\mathcal{G}$ of $\mathrm{SL}_{2}(A)$ for a general $p$-profinite $\operatorname{ring} A$, we want to have an explicit relation between $p$-profinite subgroups $\mathcal{G}$ of the form $\mathrm{SL}_{2}(A) \cap(1+X)$ for a Lie $\mathbb{Z}_{p}$-subalgebra $X \subset \mathfrak{g l}_{2}(A)$. Assuming $p>2$, Pink [P93] found a functorial explicit relation between closed subgroups in $\mathrm{SL}_{2}(A)$ and Lie subalgebras of $\mathfrak{g l}_{2}(A)$ (valid even for $A$ of characteristic $p$ ). We call subgroups of the form $\mathrm{SL}_{2}(A) \cap(1+X)$ (for a $p$-profinite Lie $\mathbb{Z}_{p}$-subalgebra $X$ of $\mathfrak{g l}_{2}(A)$ ) basic subgroups following Pink.

We prepare some notation to quote here the results in [P93]. Let $A$ be a semi-local $p$-profinite ring (not necessarily of characteristic $p$ ). Since Pink's result allows semi-local $p$-profinite algebra, we do not assume $A$ to be local in the exposition of his result. We assume $p>2$. Define maps $\Theta: \mathrm{SL}_{2}(A) \rightarrow \mathfrak{s l}_{2}(A)$ and $\zeta: \mathrm{SL}_{2}(A) \rightarrow Z(A)$ for the center $Z(A)$ of the algebra $M_{2}(A)$ by

$$
\Theta(x)=x-\frac{1}{2} \operatorname{Tr}(x)\left(\begin{array}{ll}
1 & 0 \\
0 & 1
\end{array}\right) \text { and } \zeta(x)=\frac{1}{2}(\operatorname{Tr}(x)-2)\left(\begin{array}{ll}
1 & 0 \\
0 & 1
\end{array}\right) .
$$

For each $p$-profinite subgroup $\mathcal{G}$ of $\mathrm{SL}_{2}(A)$, define $L$ by the closed additive subgroup of $\mathfrak{s l}_{2}(A)$ topologically generated by $\Theta(x)$ for all $x \in \mathcal{G}$. Then we put $C=\operatorname{Tr}(L \cdot L)$. Here $L \cdot L$ is the closed additive subgroup of $M_{2}(A)$ generated by $\{x y \mid x, y \in L\}$ for the matrix product $x y$, similarly $L^{j}$ is the closed additive subgroup generated by iterated products ( $j$ times) of elements in $L$. We then define $L_{1}=L$ and inductively $L_{j+1}=\left[L, L_{j}\right]$; so, $L_{2}=[L, L]$, where $\left[L, L_{j}\right]$ is the closed additive subgroup generated by Lie bracket $[x, y]=x y-y x$ for $x \in L$ and $y \in L_{n}$. Then by [P93, Proposition 3.1], we have

$$
[L, L] \subset L, C \cdot L \subset L, L=L_{1} \supset \cdots \supset L_{j} \supset L_{j+1} \supset \cdots \text { and } \bigcap_{j \geq 1} L_{n}=\bigcap_{j \geq 1} L^{j}=0 .
$$

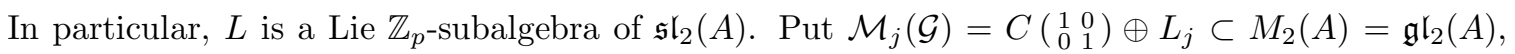
which is a closed Lie $\mathbb{Z}_{p}$-subalgebra by (3.1). Define

$$
\mathcal{H}_{j}=\left\{x \in \mathrm{SL}_{2}(A) \mid \Theta(x) \in L_{j}, \operatorname{Tr}(x)-2 \in C\right\} \text { for } j \geq 1 .
$$

If $x \in \mathcal{H}_{n}$, then $x=\Theta(x)+\zeta(x)+\left(\begin{array}{ll}1 & 0 \\ 0 & 1\end{array}\right)$; thus, $\mathcal{H}_{j} \subset \mathrm{SL}_{2}(A) \cap\left(1+\mathcal{M}_{j}(\mathcal{G})\right)$. If we pick $x \in$ $\mathrm{SL}_{2}(A) \cap\left(1+\mathcal{M}_{j}(\mathcal{G})\right)$, then $x=1+c \cdot 1+y$ with $y \in L_{n}$ and $c \in C$. Thus $\operatorname{Tr}(x)-2=2 c \in C$ and 


$$
\begin{array}{r}
\Theta(x)=\left(\begin{array}{ll}
1 & 0 \\
0 & 1
\end{array}\right)+c \cdot\left(\begin{array}{ll}
1 & 0 \\
0 & 1
\end{array}\right)+y-\frac{1}{2}(2+2 c) \cdot\left(\begin{array}{ll}
1 & 0 \\
0 & 1
\end{array}\right)=y . \text { This shows } \\
\mathcal{H}_{j}=\mathrm{SL}_{2}(A) \cap\left(1+\mathcal{M}_{j}(\mathcal{G})\right) .
\end{array}
$$

Here is a result of Pink (Theorem 3.3 combined with Theorem 2.7 both in [P93]):

Theorem 3.5 (Pink). Let the notation be as above. Suppose $p>2$, and let $A$ be a semi-local p-profinite commutative ring with identity. Take a p-profinite subgroup $\mathcal{G} \subset \mathrm{SL}_{2}(A)$. Then we have

(1) $\mathcal{G}$ is a normal closed subgroup of $\mathcal{H}_{1}$ (defined as above for $\left.\mathcal{G}\right)$,

(2) $\mathcal{H}_{n}$ is a p-profinite subgroup of $\mathrm{SL}_{2}(A)$ inductively given by $\mathcal{H}_{j+1}=\left(\mathcal{H}_{1}, \mathcal{H}_{j}\right)$ which is the closed subgroup topologically generated by commutators $(x, y)$ with $x \in \mathcal{H}_{1}$ and $y \in \mathcal{H}_{j}$,

(3) $\left\{\mathcal{H}_{j}\right\}_{j \geq 2}$ coincides with the descending central series of $\left\{\mathcal{G}_{j}\right\}_{j \geq 2}$ of $\mathcal{G}$, where $\mathcal{G}_{j+1}=\left(\mathcal{G}, \mathcal{G}_{j}\right)$ starting with $\mathcal{G}_{1}=\mathcal{G}$.

In particular, we have

(P) The topological commutator subgroup $\mathcal{G}^{\prime}$ of $\mathcal{G}$ is the subgroup given by $\mathrm{SL}_{2}(A) \cap\left(1+\mathcal{M}_{2}(\mathcal{G})\right)$ for the closed Lie subalgebra $\mathcal{M}_{2}(\mathcal{G}) \subset M_{2}(A)$ as above.

Recall that $G$ is a split (smooth) connected reductive $\mathbb{Z}_{p^{-}}$group with maximal split torus $T$. Let $G^{\prime}$ be the derived group of $G$. Now let $\mathcal{G}$ be a $p$-profinite subgroup of $G^{\prime}(A)$. Then on $\mathcal{G}_{1}=\mathcal{G} \cap \Gamma_{A}^{G}(p)$ the $\operatorname{logarithm} \sum_{m=1}^{\infty}(-1)^{m+1} \frac{(x-1)^{m}}{m}$ converges to an element $\log (x) \in \operatorname{Lie}(G)(A)$. Note that in general $\log (\mathcal{G})=\{\log (x) \mid x \in \mathcal{G}\}$ is neither an abelian group nor a Lie algebra. Fix a Borel subgroup $B_{/ \mathbb{Z}_{p}}$ of $G$ containing $T$ with $B=T U^{+}$for the unipotent radical $U^{+}$. Write $U^{-}$for the unipotent subgroup opposite to $U^{+}$; so, $T U^{-}$is the opposite Borel subgroup of $B$.

Lemma 3.6. Suppose that $A$ is a p-profinite complete local integral domain over $\mathbb{Z}_{p}$ for $p>2$. If $\mathcal{G}_{1}$ contains $\Gamma_{A}^{U^{+}}(\mathfrak{a})$ and $\Gamma_{A}^{U^{-}}(\mathfrak{b})$ for non-zero ideals $\mathfrak{a}$ and $\mathfrak{b}$ of $A$. Then $\mathcal{G}_{1}$ (and hence $\mathcal{G}$ ) contains $\Gamma_{A}^{G}\left(\mathfrak{a}^{2} \mathfrak{b}^{2}\right)$.

Proof. We write $\mathfrak{u}^{+}$(resp. $\mathfrak{u}^{-}, \mathfrak{t}$ ) for the Lie subalgebra of Lie $(G)$ corresponding to $U^{+}$(resp. $U^{-}$, $T)$. We start with the case where $G=\mathrm{SL}(2)$. Note that $\mathrm{GSpin}_{3} \cong \mathrm{SL}(2)$.

In our setting, we assume that $A$ is local. Then by the definition as above, under the notation in Theorem 3.5, we have $\log \left(\mathcal{G}_{1} \cap U^{+}(A)\right)=\mathcal{M}_{1}\left(\mathcal{G}_{1}\right) \cap U^{+}(A)$ and $\log \left(\mathcal{G}_{1} \cap U^{-}(A)\right)=\mathcal{M}_{1}\left(\mathcal{G}_{1}\right) \cap U^{-}(A)$. Thus $\mathcal{M}_{1}\left(\mathcal{G}_{1}\right)$ contains $\mathfrak{u}^{+}(\mathfrak{a})=\left\{\left(\begin{array}{cc}0 & a \\ 0 & 0\end{array}\right) \mid a \in \mathfrak{a}\right\}$ and $\mathfrak{u}^{-}\left(\mathfrak{a}^{\prime}\right)=\left\{\left(\begin{array}{cc}0 & 0 \\ a & 0\end{array}\right) \mid a \in \mathfrak{a}^{\prime}\right\}$. Thus $\left[\mathfrak{u}^{+}(\mathfrak{a}), \mathfrak{u}^{-}\left(\mathfrak{a}^{\prime}\right)\right] \supset$ $\mathfrak{t}\left(\mathfrak{a} \mathfrak{a}^{\prime}\right):=\left\{\left(\begin{array}{cc}a & 0 \\ 0 & -a\end{array}\right) \mid a \in \mathfrak{a a}^{\prime}\right\}$ is contained in $\mathcal{M}_{1}\left(\mathcal{G}^{\prime}\right)$. In other words, $\mathcal{M}_{1}\left(\mathcal{G}_{1}\right) \supset \mathcal{M}_{1}\left(\Gamma_{A}^{G}\left(\mathfrak{a} \mathfrak{a}^{\prime}\right)\right)$. This implies $\mathcal{G}_{1}^{\prime}$ contains the derived group of $\Gamma_{A}^{G}\left(\mathfrak{a} \mathfrak{a}^{\prime}\right)$ which contains $\Gamma_{A}^{G}\left(\mathfrak{a}^{2} \mathfrak{a}^{2}\right)$. This finishes the proof for $n=1$.

For general $G$, we pick a positive root $\alpha$ of $T$ with root subgroup $U_{\alpha} \subset U^{+}$. Then we have an embedding $i_{\alpha}$ : SL(2) $\hookrightarrow G$ sending the upper (resp. lower) triangular unipotent subgroup of SL(2) to $U_{\alpha}$ (resp. $U_{-\alpha} \subset U^{-}$). Applying the argument in the case of $\operatorname{SL}(2)$ to $\mathcal{G}_{\alpha}:=i_{\alpha}^{-1}(\mathcal{G})$, we have $\mathcal{G}_{\alpha} \supset \Gamma_{A}^{\mathrm{SL}(2)}\left(\mathfrak{a}^{2} \mathfrak{b}^{2}\right)$. It is easy to see that $\left\{i_{\alpha}\left(\Gamma_{A}^{\mathrm{SL}(2)}\left(\mathfrak{a}^{2} \mathfrak{b}^{2}\right)\right)\right\}_{\alpha}$ generates $\Gamma_{A}^{G}\left(\mathfrak{a}^{2} \mathfrak{b}^{2}\right)$, and hence the desired assertion holds.

3.6. $\Lambda$-fullness for the endoscopic cases. We return to the setting of Section 3.4. We now study $\Lambda$-fullness in the one variable case of $\Lambda=\Lambda_{1}=\mathbb{Z}_{p}[[T]]$ in the endoscopic cases (Y), (K), (C) and (S) below. In each endoscopic case, we have a factor GL(2) of $G$. Even if the component of the Hecke algebra has two variables, the Galois image of the projected factor falls in $\mathrm{GL}_{2}(\mathbb{I})$ for $\mathbb{I}$ finite over $\Lambda$ (for a suitable quotient $\Lambda$ of $\Lambda_{2}$ ). Thus we do not lose any generality assuming that $\mathbb{I}$ is finite over one variable $\Lambda$. To describe the quotient map $\Lambda_{2} \rightarrow \Lambda$ explicitly, we normalize first $\Lambda_{2}=\mathbb{Z}_{p}\left[\left[T_{1}, T_{2}\right]\right]$ so that the Galois image to contain

$$
\mathcal{T}=\left\{\operatorname{diag}\left[\left(t_{1} t_{2}\right)^{s}, t_{1}^{s}, t_{2}^{s}, 1\right] \in T\left(\Lambda_{2}\right) \mid s \in \mathbb{Z}_{p}, t_{j}=1+T_{j}\right\}
$$

as the wild $p$-inertia image. Then we normalize the Galois representation attached an $\mathbb{I}$-adic ordinary Hecke eigenform form as follows depending on cases for $\rho=\rho_{\mathbb{I}}$.

- In Case (Y), we write $\rho \cong \phi \times \varphi$ with $\phi(\sigma)=\left(\begin{array}{cc}a(\sigma) & b(\sigma) \\ c(\sigma) & d(\sigma)\end{array}\right), \varphi(\sigma)=\left(\begin{array}{cc}\alpha(\sigma) & \beta(\sigma) \\ \gamma(\sigma) & \delta(\sigma)\end{array}\right)$ and

$$
\rho(\sigma)=\left(\begin{array}{cccc}
a(\sigma) & 0 & 0 & b(\sigma) \\
0 & \alpha(\sigma) & \beta(\sigma) & 0 \\
0 & \gamma(\sigma) & \delta(\sigma) & 0 \\
c(\sigma) & 0 & 0 & d(\sigma)
\end{array}\right) \quad \text { and } \Lambda=\Lambda_{2} /\left(t_{1} t_{2}-t_{1}\right) \text { with } t_{1} \mapsto t=1+T .
$$


- In Case $(\mathrm{K}), \rho \cong \chi \oplus \chi^{\prime} \oplus \phi$ with $\phi(\sigma)=\left(\begin{array}{ll}a(\sigma) & b(\sigma) \\ c(\sigma) & d(\sigma)\end{array}\right)$ and

$$
\rho(\sigma)=\left(\begin{array}{cccc}
a(\sigma) & 0 & 0 & b(\sigma) \\
0 & \chi(\sigma) & 0 & 0 \\
0 & 0 & \chi^{\prime}(\sigma) & 0 \\
c(\sigma) & 0 & 0 & d(\sigma)
\end{array}\right) \quad \text { and } \Lambda=\Lambda_{2} /\left(t_{1}-t_{2}\right) \text { with } t_{1} t_{2} \mapsto t=1+T \text {. }
$$

- In Case $(\mathrm{S}), \rho \cong\left(\phi^{\iota} \otimes \chi\right) \oplus \phi$; i.e.,

$$
\rho(\sigma)=\left(\begin{array}{cc}
\chi(\sigma) s^{t} \phi(\sigma)^{\iota} s^{-1} & 0 \\
0 & \phi(\sigma)
\end{array}\right) \text { and } \Lambda=\Lambda_{2} /\left(t_{1}-1\right) \text { with } t_{2} \mapsto t=1+T .
$$

- In Case (C), and $\Lambda=\Lambda_{2} /\left(t_{1}-t_{2}^{2}\right)$ with $t_{2} \mapsto t=1+T$.

Suppose

(y) In Case $(\mathrm{Y}), n=2, \operatorname{det} \varphi=\operatorname{det} \rho=\nu \rho$ and for $\sigma \in I_{p}$,

$$
\phi(\sigma)=\left(\begin{array}{cc}
\left(t_{1} t_{2}\right)^{\log _{p}\left(\epsilon(\sigma) / \log _{p}(1+p)\right.} & * \\
0 & 1
\end{array}\right), \varphi(\sigma)=\left(\begin{array}{cc}
t_{1}^{\log _{p}\left(\epsilon(\sigma) / \log _{p}(1+p)\right.} & * \\
0 & t_{2} \log _{p}\left(\epsilon(\sigma) / \log _{p}(1+p)\right.
\end{array}\right),
$$

and $\phi$ has values in a finite extension $\mathbb{I}_{\phi} \subset \mathbb{I}$ of $\Lambda_{\phi}=\mathbb{Z}_{p}\left[\left[T_{\phi}\right]\right]$ for $T_{\phi}=t_{1} t_{2}-1$ and $\varphi \otimes \kappa^{-1}$ has values in a finite extension $\mathbb{I}_{\varphi} \subset \mathbb{I}$ of $\Lambda_{\varphi}=\mathbb{Z}_{p}\left[\left[T_{\varphi}\right]\right]$ for $T_{\varphi}=t_{1} t_{2}^{-1}-1$, where $\kappa: G_{\mathbb{Q}} \rightarrow \Lambda_{\varphi}^{\times}$ is a character such that $\kappa(\sigma)=t_{2}^{\log _{p}\left(\epsilon(\sigma) / \log _{p}(1+p)\right.}$.

(k) In Case $(\mathrm{K}), n=2$ and for $\sigma \in I_{p}, \chi(\sigma)=t_{1}^{\log _{p}\left(\epsilon(\sigma) / \log _{p}(1+p)\right.}, \chi^{\prime}(\sigma)=t_{2}^{\log _{p}\left(\epsilon(\sigma) / \log _{p}(1+p)\right.}$ and

$$
\phi(\sigma)=\left(\begin{array}{cc}
\left(t_{1} t_{2}\right)^{\log _{p}\left(\epsilon(\sigma) / \log _{p}(1+p)\right.} * \\
0 & 1
\end{array}\right),
$$

and $\phi$ has values in a finite extension $\mathbb{I}_{\phi} \subset \mathbb{I}$ of $\Lambda_{\phi}=\mathbb{Z}_{p}\left[\left[T_{\phi}\right]\right]$ for $T_{\phi}=t_{1} t_{2}-1$.

(s) In Case $(\mathrm{S}), n=2$ and $\chi(\sigma)=t_{1}^{\log _{p}\left(\epsilon(\sigma) / \log _{p}(1+p)\right.}$ and $\phi(\sigma)=\left(\begin{array}{cc}t_{2}^{\log _{p}\left(\epsilon(\sigma) / \log _{p}(1+p)\right.} * \\ 0\end{array}\right)$ for $\sigma \in I_{p}$, and $\phi$ has values in a finite extension $\mathbb{I}_{\phi} \subset \mathbb{I}$ of $\Lambda_{\phi}=\mathbb{Z}_{p}\left[\left[T_{\phi}\right]\right]$ for $T_{\phi}=t_{2}-1$.

(c) In Case (C), $n=1$ and for $\sigma \in I_{p}$,

$$
\rho(\sigma)=\left(\begin{array}{cccc}
t_{1}{ }^{3 \log _{p}\left(\epsilon(\sigma) / \log _{p}(1+p)\right.} & * & * & * \\
0 & t_{1}{ }^{2 \log _{p}\left(\epsilon(\sigma) / \log _{p}(1+p)\right.} & * & * \\
0 & 0 & t_{1} \log _{p}\left(\epsilon(\sigma) / \log _{p}(1+p)\right. & * \\
0 & 0 & 0 & 1
\end{array}\right) .
$$

Note here $\mathrm{GSpin}_{3} \cong \mathrm{SL}(2)$ and $\mathrm{GSpin}_{5}=\mathrm{GSp}(4)$ over $\mathbb{Z}_{p}$ (for $p$ odd). Let $\mathbb{I}$ be a finite flat normal extension of $\Lambda$ as before, and $\rho_{\mathbb{I}}: \operatorname{Gal}(\overline{\mathbb{Q}} / \mathbb{Q}) \rightarrow \operatorname{GSpin}_{2 n+1}(\mathbb{I})$ be a Galois representation. Suppose

(G0) $G \supset \mathcal{T}$; so, $\operatorname{rank} G=\operatorname{rank} \mathrm{GSpin}_{2 n+1}$,

(G1) there exists a point $P \in \operatorname{Spec}(\Lambda)\left(\overline{\mathbb{Q}}_{p}\right)$ such that $\rho_{P}=\left(\rho_{\mathbb{I}} \bmod P\right)$ is $\mathbb{Z}_{p}$-full with respect to $G$, regarding $\mathbb{I} / P \mathbb{I}$ as a $\mathbb{Z}_{p}$-algebra.

(G2) the semi-simplification of $\rho_{\mathbb{I}}$ restricted to the wild $p$-inertia group $I_{p}^{w}$ is isomorphic to the diagonal representation $\operatorname{diag}\left[\boldsymbol{\kappa}^{2 n-1}, \boldsymbol{\kappa}^{2 n-2}, \cdots, 1\right]$ for the character $\boldsymbol{\kappa}: I_{p}^{w} \rightarrow \Lambda^{\times}$such that $\kappa\left(\left[1+p, \mathbb{Q}_{p}\right]\right)=t=1+T$.

(G3) $\left(\nu \circ \rho_{\mathbb{I}}\right) / \boldsymbol{\kappa}^{n(2 n-1)}$ has finite order.

(G4) We have $\operatorname{diag}\left[\boldsymbol{\kappa}^{2 n-1}, \boldsymbol{\kappa}^{2 n-2}, \cdots, 1\right]$ in $\rho_{\mathbb{I}}\left(I_{p}^{w}\right)$.

Conjecture 3.7. Assume $p>2 n-1>2$ in addition to (G0-4). Then $\rho_{\mathbb{I}}$ is $\Lambda$-full with respect to $G$.

Theorem 3.8. Suppose that $n=1,2$ and that we are not in Case (F) nor in Case (T) (so we are in the endoscopic cases). Let $\rho_{\mathbb{I}}$ be associated to an $\mathbb{I}$-adic ordinary Hecke eigen cusp form. Assume $\mathbb{Z}_{p}$-regularity if $\mathbb{I} \neq \Lambda$ or in Case $(\mathrm{Y})$. Then the above conjecture holds.

We prove below the $\Lambda$-fullness including $p=3$.

Proof. When $n=1$, this follows from [H13b, Theorem I]. If $\mathbb{I}=\Lambda$, by [Z14], we have a non-trivial upper unipotent element in $\rho_{\mathbb{I}}\left(I_{p}\right)$ and also a lower one; so, (G4) is actually not necessary if $\mathbb{I}=\Lambda$. If $\mathbb{I} \neq \Lambda, \mathbb{Z}_{p}$-regularity is used in $[\mathrm{H} 13 \mathrm{~b}]$ to show non-trivial unipotents can be found in $\mathrm{SL}_{2}(\Lambda)$ (not just in $\mathrm{SL}_{2}(\mathbb{I})$ shown in $\left.[\mathrm{Z} 14]\right)$. Since we are in the cases different from $(\mathrm{F})$ and $(\mathrm{T}), G^{\prime}$ is either split SL(2) or SL(2) $\times \mathrm{SL}(2)$. This implies that, by GL(2)-theory, we can find an arithmetic prime $P \in \operatorname{Spec}(\Lambda)$ (sufficiently regular) such that $\rho:=\rho_{\mathfrak{P}}$ for every prime $\mathfrak{P} \in \operatorname{Spec}(\mathbb{I})$ above $P$ belongs 
to the same case except for Case (F). Then by Proposition 3.4 applied to $\rho, \rho_{j, \mathfrak{p}}$ is $\mathbb{Z}_{p}$-full, and hence the argument in $[\mathrm{H} 13 \mathrm{~b}]$ works.

Since we have one simple component SL(2) except for Case $(\mathrm{Y})$, if we are not in Cases (Y) and $(\mathrm{F})$, there is no difference in the argument proving the theorem from the one given in [H13b]. Thus we give more details in Case $(\mathrm{Y})$. In this case, $\rho_{\mathbb{I}}$ has values in $\mathrm{GL}_{2}(\mathbb{I}) \times \mathrm{GL}_{2}(\mathbb{I})$. We write $\rho_{L}, \rho_{R}: \operatorname{Gal}(\overline{\mathbb{Q}} / \mathbb{Q}) \rightarrow \mathrm{GL}_{2}(\mathbb{I})$ for the projection of $\rho_{\mathbb{I}}$ to the left and right factor $\mathrm{GL}_{2}(\mathbb{I})$. Each projection $\rho_{\mathbb{I}, j}(j=1,2)$ to each simple component $\mathrm{GL}(2)$ is $\Lambda$-full by the argument in [H13b]. Pink's theory can be also applied to semi-local $p$-profinite rings, like $A=\mathbb{I} \oplus \mathbb{I}$. Put $\mathcal{G}=\operatorname{Im}\left(\rho_{\mathbb{I}}\right) \cap \Gamma_{\mathbb{I} \oplus \mathbb{I}}^{\mathrm{SL}(2)}\left(\mathfrak{m}_{\mathbb{I}}\right)$ for the maximal ideal $\mathfrak{m}_{\mathbb{I}}$. and $\mathcal{G}_{P}=\operatorname{Im}\left(\rho_{P}\right) \cap \Gamma_{\mathbb{I} / P \oplus \mathbb{S} / P}^{\mathrm{SL}(2)}\left(\mathfrak{m}_{\mathbb{I} / P}\right)$. Since the reduction map modulo $P$ is onto for Pink's Lie algebra, we have a surjective morphism $\mathcal{M}_{j}(\mathcal{G}) \rightarrow \mathcal{M}_{j}\left(\mathcal{G}_{P}\right)$. Adding the superscript "+" (resp. "-", "0") to Pink's Lie algebra, we indicates the upper nilpotent subalgebra (resp. the lower nilpotent subalgebra, the diagonal subalgebra). Then by $\mathbb{Z}_{p}$-regularity, $\mathcal{M}_{j}(\mathcal{G})=$ $\mathcal{M}_{j}^{+}(\mathcal{G}) \oplus \mathcal{M}_{j}^{0}(\mathcal{G}) \oplus \mathcal{M}_{j}^{-}(\mathcal{G})$ and the same for $\mathcal{M}_{j}\left(\mathcal{G}_{P}\right)$. Thus the reduction map modulo $P$ induces a sujection $\mathcal{M}_{j}^{*}(\mathcal{G}) \rightarrow \mathcal{M}_{j}^{*}\left(\mathcal{G}_{P}\right)$ for $*= \pm, 0$.

Let $1_{L}$ (resp. $1_{R}$ ) be the idempotent of the left (resp. the right) factor $\mathbb{I}$ of $A$. Since $\mathcal{G}_{P}$ is $\mathbb{Z}_{p}$-full, for $\mathcal{M}_{j}\left(\mathcal{G}_{P}\right), 1_{L} \mathcal{M}_{j}^{*}\left(\mathcal{G}_{P}\right) \neq 0$ and $1_{R} \mathcal{M}_{j}^{*}\left(\mathcal{G}_{P}\right) \neq 0$ for $*= \pm, 0$. Take $g \in \operatorname{Im}\left(\rho_{\mathbb{I}}\right)$ giving the $\mathbb{Z}_{p}$-regularity, $A d(g)$ acts on $1_{L} \mathcal{M}_{j}^{ \pm}\left(\mathcal{G}_{P}\right) \cap \mathcal{M}_{j}^{ \pm}\left(\mathcal{G}_{P}\right)$ and $1_{R} \mathcal{M}_{j}^{ \pm}\left(\mathcal{G}_{P}\right) \cap \mathcal{M}_{j}^{ \pm}\left(\mathcal{G}_{P}\right)$ by the different set of eigenvalues. Thus $1_{?} \mathcal{M}_{j}^{ \pm}\left(\mathcal{G}_{P}\right) \cap \mathcal{M}_{j}^{ \pm}\left(\mathcal{G}_{P}\right) \neq 0$ for $?=L, R$. We have eigenspace decompositions under the action of $\operatorname{Ad}(g): \mathcal{M}_{j}^{ \pm}\left(\mathcal{G}_{P}\right)=\left(1_{L} \mathcal{M}_{j}^{ \pm}\left(\mathcal{G}_{P}\right) \cap \mathcal{M}_{j}^{ \pm}\left(\mathcal{G}_{P}\right)\right) \oplus\left(1_{L} \mathcal{M}_{j}^{ \pm}\left(\mathcal{G}_{P}\right) \cap \mathcal{M}_{j}^{ \pm}\left(\mathcal{G}_{P}\right)\right)$ and $\mathcal{M}_{j}^{ \pm}(\mathcal{G})=\left(1_{L} \mathcal{M}_{j}^{ \pm}(\mathcal{G}) \cap \mathcal{M}_{j}^{ \pm}(\mathcal{G})\right) \oplus\left(1_{L} \mathcal{M}_{j}^{ \pm}(\mathcal{G}) \cap \mathcal{M}_{j}^{ \pm}(\mathcal{G})\right)$. This implies $1_{L} \mathcal{M}_{j}^{ \pm}(\mathcal{G}) \cap \mathcal{M}_{j}^{ \pm}(\mathcal{G}) \neq 0$ and $1_{R} \mathcal{M}_{j}^{ \pm}(\mathcal{G}) \cap \mathcal{M}_{j}^{ \pm}(\mathcal{G}) \neq 0$ as the modulo $P$ reduction map of $1_{?} \mathcal{M}_{j}^{ \pm}(\mathcal{G}) \cap \mathcal{M}_{j}^{ \pm}(\mathcal{G})$ to $1_{?} \mathcal{M}_{j}^{ \pm}\left(\mathcal{G}_{P}\right) \cap$ $\mathcal{M}_{j}^{ \pm}\left(\mathcal{G}_{P}\right)$ is surjective for $?=L, R$. This implies that $\left(1_{?} \mathcal{M}_{j}^{ \pm}(\mathcal{G}) \cap \mathcal{M}_{j}^{ \pm}(\mathcal{G})\right)=1_{?} \mathcal{M}_{j}^{ \pm}(\mathcal{G})$ for $?=L, R$ and for all $j>0$. After replacing $\rho_{\mathbb{I}}$ by a conjugate under $G(Q(\mathbb{I}))$, by [H13b, Lemma 2.9], that $\operatorname{Im}\left(\rho_{L}\right) \supset \Gamma_{\Lambda}^{\mathrm{SL}(2)}\left(\mathfrak{c}_{L}\right)$ and $\operatorname{Im}\left(\rho_{R}\right) \supset \Gamma_{\Lambda}^{\mathrm{SL}(2)}\left(\mathfrak{c}_{R}\right)$ for non-zero $\Lambda$-ideals $\mathfrak{c}_{L}$ and $\mathfrak{c}_{R}$. In particular, by Lemma 3.6, we get $\operatorname{Im}\left(\rho_{\mathbb{I}}\right) \supset \Gamma_{A}^{\mathrm{SL}(2)}(\mathfrak{c})$ for $\mathfrak{c}=\mathfrak{c}_{L}^{2} \cap \mathfrak{c}_{R}^{2}$ as desired.

3.7. $\mathbb{Z}_{p}\left[\left[S_{1}\right]\right] \oplus \mathbb{Z}_{p}\left[\left[S_{2}\right]\right]$-fullness in Case $(\mathbf{Y})$. We can think of fullness property in two variable setting. Case (F) will be treated in the following section in the general setting of GSpin representations as $\mathrm{GSpin}_{5} \cong \mathrm{GSp}(4)$. In the cases other than (F) and (Y), the abelian part consumes one variable out of the two, we do not have a properly two variable Galois representation $\rho_{\mathbb{I}}$. Thus we assume that there exists a finite extension $k / \mathbb{Q}$ such that $\rho:=\left.\rho_{\mathbb{I}}\right|_{\text {Gal }(\overline{\mathbb{Q}} / k)}$ has values in

$$
G_{2}=\{(x, y) \in \mathrm{GL}(2) \times \mathrm{GL}(2) \mid \operatorname{det}(x)=\operatorname{det}(y)\} .
$$

We suppose that $G_{2}$ is embedded into $\operatorname{GSp}(4)$ in the following way:

$$
G_{2} \ni\left(\left(\begin{array}{ll}
a & b \\
c & d
\end{array}\right),\left(\begin{array}{ll}
\alpha & \beta \\
\gamma & \delta
\end{array}\right)\right) \mapsto\left(\begin{array}{cccc}
a & 0 & 0 & b \\
0 & \alpha & \beta & 0 \\
0 & \gamma & 0 & 0 \\
c & 0 & 0 & d
\end{array}\right) \in \operatorname{GSp}(4)
$$

As before, we suppose that $\rho\left(I_{p}^{w}\right)$ for the wild $p$-inertia group $I_{p}^{w}$ contains the following split torus

$$
\mathcal{T}=\left\{\operatorname{diag}\left[t_{1}^{s} t_{2}^{s}, t_{1}^{s}, t_{2}^{s}, 1\right] \in T \mid s \in \mathbb{Z}_{p}\right\}
$$

for $t_{j}=1+T_{j}$ and $\Lambda_{2}=\mathbb{Z}_{p}\left[\left[T_{1}, T_{s}\right]\right]$. We make the following variable change $s_{1}=t_{1} t_{2}$ and $s_{2}=t_{1} / t_{2}$; so, $\mathbb{Z}_{p}\left[\left[S_{1}, S_{2}\right]\right]=\Lambda_{2}$ for $S_{j}=s_{j}-1$. We put $A_{j}=\mathbb{Z}_{p}\left[\left[S_{j}\right]\right] ;$ so, $\Lambda_{2}=\mathbb{Z}_{p}\left[\left[S_{1}, S_{2}\right]\right]=A_{1} \widehat{\otimes}_{\mathbb{Z}_{p}} A_{2}$.

Proposition 3.9. Let $\rho_{\mathbb{I}}$ is associated to I-adic ordinary Hecke eigenform on $\mathrm{GSp}(4)$ for an algebra II finite torsion-free over $\Lambda_{2}$. Suppose $\mathbb{Z}_{p}$-regularity for $\rho_{\mathbb{I}}$ and that for an arithmetic point $P \in$ $\operatorname{Spec}\left(\Lambda_{2}\right)\left(\overline{\mathbb{Q}}_{p}\right)$ and for all primes $\mathfrak{P} \in \operatorname{Spec}(\mathbb{I})\left(\overline{\mathbb{Q}}_{p}\right)$ above $P, \rho_{\mathfrak{P}}=\rho \bmod \mathfrak{P}$ belongs to Case $(\mathrm{Y})$ in Section 3.4. Then, if $p \geq 3$, replacing $\rho$ by a conjugate of $\rho$ by an element in $\mathrm{GL}_{2}\left(Q\left(A_{1}\right)\right) \times$ $\mathrm{GL}_{2}\left(Q\left(A_{2}\right)\right)$ if necessary, there exist non-zero $A_{j}$-ideals $\mathfrak{c}_{j}$ such that $\operatorname{Im}(\rho)$ contains

$$
\Gamma_{A_{1}}^{\mathrm{SL}(2)}\left(\mathfrak{c}_{1}\right) \times \Gamma_{A_{2}}^{\mathrm{SL}(2)}\left(\mathfrak{c}_{2}\right) \subset \mathrm{SL}_{2}\left(A_{1}\right) \times \mathrm{SL}_{2}\left(A_{2}\right) \subset G_{2}\left(\Lambda_{2}\right) .
$$

Proof. Let $\{i, j\}=\{1,2\}$. We can write $P=P_{i} \otimes P_{j}$ for $P_{\text {? }} \in \operatorname{Spec}\left(A_{\text {? }}\right)(\overline{\mathbb{Q}})$ for $?=i, j$. Consider the projection $P_{i} \times$ id $: \Lambda_{2}:=A_{i} \widehat{\otimes}_{\mathbb{Z}_{p}} A_{j} \rightarrow W_{i} \otimes_{\mathbb{Z}_{p}} A_{j}=W_{i}\left[\left[S_{j}\right]\right]$ for $W_{i}=A_{i} / P_{i}$. Take a prime $\widetilde{\mathfrak{P}}_{i} \in \operatorname{Spec}(\mathbb{I})$ above $\operatorname{Ker}\left(P_{i} \times \mathrm{id}\right.$ ). Let $\rho_{j}=\rho \bmod \widetilde{\mathfrak{P}}_{i}$. Then by Theorem 3.8 (or more precisely, 
by the same argument proving the theorem), replacing $\rho_{j}$ by its conjugate under an element of $\mathrm{GL}_{2}\left(Q\left(A_{j}\right)\right), \operatorname{Im}\left(\rho_{j}\right)$ contains $\Gamma_{A_{j}}^{\mathrm{SL}(2)}\left(\mathfrak{a}_{j}\right)$ for a non-zero $A_{j}$-ideal $\mathfrak{a}_{j}$.

Put $\mathcal{G}=\operatorname{Im}(\rho) \cap \Gamma_{\Lambda_{2}}^{\mathrm{Sp}(4)}\left(\Lambda_{2}\right)$, and consider Pink's Lie algebra $\mathcal{M}_{2}(\mathcal{G})$ with respect to $A=A_{1} \oplus A_{2}$ regarding $\mathrm{SL}_{2}\left(A_{1}\right) \times \mathrm{SL}_{2}\left(A_{2}\right) \subset G_{2}\left(\Lambda_{2}\right)$ as $\mathrm{SL}_{2}\left(A_{1} \oplus A_{2}\right)$. Write $\mathfrak{u}_{j}^{+}$for the upper nilpotent Lie algebra of the $j$-th component of $\operatorname{SL}(2) \times \mathrm{SL}(2)$, and let $\mathfrak{u}_{j}^{-}$be the opposite algebra of $\mathfrak{u}_{j}^{+}$. By $\mathbb{Z}_{p}$-regularity of $\rho$, we have $\sigma \in \operatorname{Gal}(\overline{\mathbb{Q}} / K)$ normalizing $\mathcal{G}$ whose adjoint action $\operatorname{Ad}(\rho(\sigma)$ has distinct $\mathbb{Z}_{p}$-eigenvalues on $\mathfrak{u}_{j}^{ \pm}$for $j=1,2$.

Since $\operatorname{Im}\left(\rho_{j}\right)$ contains $\Gamma_{A_{j}}^{\mathrm{SL}(2)}\left(\mathfrak{a}_{j}\right), \mathfrak{n}_{j}^{ \pm}:=\mathcal{M}_{2}(\mathcal{G}) \cap \mathfrak{u}_{j}^{ \pm}$is non-trivial. By the adjoint action of $\mathcal{T}, \mathfrak{n}_{j}^{ \pm}$is a $A_{j}$-module. Then by [H13b, Lemma 2.9] applied to each projection of $\rho$ to GL(2), we conclude a suitable conjugate of $\rho$ has image containing the product of congruence subgroups as in the proposition.

\section{Fullness of the Galois image in general Spin groups over Iwasawa algebras}

4.1. Spin groups. Let us first recall the definition and the basic properties of the $\mathbb{Z}\left[\frac{1}{2}\right]$-group scheme $\operatorname{GSpin}_{2 n+1}$ of spinorial similitudes. Consider the quadratic form $Q(\underline{x})=2 x_{1} x_{2 n+1}+2 x_{2} x_{2 n}+\ldots+$ $2 x_{n-1} x_{n+1}+x_{n}^{2}$ on $L=\bigoplus_{i=1}^{2 n+1} \mathbb{Z}\left[\frac{1}{2}\right] \cdot e_{i}$. Let $C=C(L, Q)$ be the Clifford algebra associated to $(L, Q)$ and $C=C^{+} \oplus C^{-}$be its decomposition as $\mathbb{Z} / 2 \mathbb{Z}$-graded algebra into even and odd Clifford elements ; $C^{+}$is a subalgebra. The main (anti-)involution $*$ sends a pure element $v_{1} \cdot \ldots \cdot v_{r}$ to $v_{r} \cdot \ldots \cdot v_{1}$. It leaves $C^{+}$stable ; the module $C$, resp. $C^{+}$, is locally free of rank $2^{2 n+1}$, resp. $2^{2 n}$; $L$ embeds in $C^{-}$by $v \mapsto v$. Then $\operatorname{GSpin}_{2 n+1}=\left\{x \in\left(C^{+}\right)^{\times} ; x \cdot L \cdot x^{*}=L\right\}$ and $\operatorname{Spin}_{2 n+1}=\{x \in$ $\left.\left(C^{+}\right)^{\times} ; x \cdot L \cdot x^{*}=L, x^{*} x=1\right\}$. We have two exact sequences of group schemes

$$
1 \rightarrow \operatorname{Spin}_{2 n+1} \rightarrow \operatorname{GSpin}_{2 n+1} \stackrel{\mu}{\rightarrow} \mathbb{G}_{m} \rightarrow 1
$$

by $\mu: x \mapsto x^{*} x$, and

$$
1 \rightarrow \mathbb{G}_{m} \rightarrow \operatorname{GSpin}_{2 n+1} \rightarrow \mathrm{SO}(L, Q) \rightarrow 1
$$

by $x \mapsto\left(v \mapsto x v x^{-1}\right)$. Since 2 is invertible, we can write $L=W \oplus W^{\vee} \oplus U$ where $W$, resp. $W^{\vee}$, is the standard lagrangian generated by $e_{1}, \ldots, e_{n}$, resp. its dual, identified to the direct factor generated by $e_{n+2}, \ldots, e_{2 n+1}$, and $U=\mathbb{Z} e_{n+1}$. There is a natural isomorphism $C^{+} \cong \operatorname{End}\left(\bigwedge^{\bullet} W\right.$ ) (FultonHarris, Lemma 20.16 , the proof works with $\mathbb{C}$ replaced by $\left.\mathbb{Z}\left[\frac{1}{2}\right]\right)$; by restriction to GSpin, it induces the spin representation spin: GSpin $\rightarrow \mathrm{GL}\left(\bigwedge^{\bullet} W\right)=\mathrm{GL}_{2^{n}}$. Let $T_{\text {spin }}$ be the diagonal maximal torus of $\mathrm{GSpin}_{2 n+1}$, and put $T_{\text {spin }}^{\prime}=T_{\text {spin }} \cap \operatorname{Spin}_{2 n+1}$. We denote by $\left(t_{1}, \ldots, t_{n} ; \mu\right) \mapsto\left[t_{1}, \ldots, t_{n} ; \mu\right]$ the standard isomorphism $\mathbb{G}_{m}^{n} \times \mathbb{G}_{m} \cong T_{\text {spin }}$, with $\mu\left(\left[t_{1}, \ldots, t_{n} ; \mu\right]\right)=\mu$. In particular $\left(t_{1}, \ldots, t_{n}\right) \mapsto$ $\left[t_{1}, \ldots, t_{n} ; 1\right]$ is an isomorphism $\mathbb{G}_{m}^{n} \cong T_{\text {spin }}^{\prime}$. We define the upper, resp. lower, triangular Borel subgroup $B_{\text {spin }}=T_{\text {spin }}^{\prime} \cdot U_{\text {spin }}$ resp. ${ }^{t} B_{\text {spin }}=T_{\text {spin }}^{\prime}{ }^{t} U_{\text {spin }}$, of $\operatorname{Spin}_{2 n+1}$ as $\operatorname{spin}^{-1}\left(B_{2^{n}}\right)$,resp. $\operatorname{spin}^{-1}\left({ }^{t} B_{2^{n}}\right)$, where $B_{2^{n}}=T_{2^{n}} \cdot U_{2^{n}}$ denotes the upper triangular Borel subgroup of $\mathrm{GL}_{2^{n}}$.

The triple $\left(\mathrm{GSpin}_{2 n+1}, B_{\text {spin }}, T_{\text {spin }}\right)$ identifies to the dual $(\widehat{G}, \widehat{B}, \widehat{T})$ of the triple $(G, B, T)$ (see [MT02, Sect.3.2]). In particular, the parametrization of (the semisimple part of) the standard torus $T_{\text {spin }}^{\prime}=\widehat{T}$ of $\widehat{G}=\operatorname{GSpin}_{2 n+1}$ by $\left[t_{1}, \ldots, t_{n} ; 1\right]$ can be written as $\prod_{i}\left[e_{i}-\frac{1}{2} f\right]\left(t_{i}\right)$ via $\widehat{T}=\mathbb{G}_{m} \otimes X^{*}(T)$, where $e_{i}:\left(x_{1}, \ldots, x_{n}, \nu x_{n}^{-1}, \ldots, \nu x_{1}^{-1}\right) \mapsto x_{i}$ and $f:\left(x_{1}, \ldots, x_{n}, \nu x_{n}^{-1}, \ldots, \nu x_{1}^{-1}\right) \mapsto \nu$.

4.2. Genus $n$ Hida families. Let $n \geq 1$ and $d=\frac{n(n+1)}{2}$. Let $\mathfrak{H}$ be the genus $n$ Siegel half space ; it is a $d$-dimensional hermitian symmetric domain. Let $G=\mathrm{GSp}_{2 n}$ with its standard Borel subgroup $B$ and its diagonal torus $T$ as before. For any torsion-free compact open subgroup $K \subset G\left(\mathbb{A}^{\infty}\right)$, we form the $d$-dimensional Shimura Siegel variety $X_{K}=G(\mathbb{Q}) \backslash\left(\mathfrak{H} \times G\left(\mathbb{A}^{\infty}\right) / K\right)$. These are nonconnected $d$-dimensional complex varieties; the inclusions $K^{\prime} \subset K$ induce a projective system of finite coverings $\pi_{K^{\prime}, K}: X_{K^{\prime}} \rightarrow X_{K}$. These varieties and morphisms are algebraic and admit quasiprojective canonical models over $\mathbb{Q}$ which we still denote by $X_{K}$ and $\pi_{K^{\prime}, K}$. Let $\lambda \in X^{*}(T)$ be a dominant weight for $(G, B, T)$ and $V_{\lambda}$ the irreducible representation of $G$ over $\mathbb{Q}$ associated to $\lambda$. It defines a projective system of étale local systems $V_{\lambda, K}\left(\overline{\mathbb{Q}}_{p}\right)$ over the $X_{K}$ 's : $\pi_{K^{\prime}, K}^{*} V_{\lambda, K}\left(\overline{\mathbb{Q}}_{p}\right)=$ $V_{\lambda, K^{\prime}}\left(\overline{\mathbb{Q}}_{p}\right)$. Let $M \geq 3$ and $K(M)$ be the level $M$ principal congruence subgroup of $G(\widehat{\mathbb{Z}})$. Let us fix an odd prime $p$ not dividing $M$ and consider the subgroups $I_{m}^{+} \subset I_{m}$ of $K_{p}=G\left(\mathbb{Z}_{p}\right)$ where 
$I_{m}=\left\{g \in K_{p} ; g\left(\bmod p^{m}\right) \in B\left(\mathbb{Z} / p^{m} \mathbb{Z}\right)\right\}$ and $I_{m}^{+}=\left\{g \in K_{p} ; g\left(\bmod p^{m}\right) \in N\left(\mathbb{Z} / p^{m} \mathbb{Z}\right)\right\}$ denotes its (normal) pro- $p$-Sylow. Let $K_{m}=K(M)^{p} \times I_{m}^{+}$. Consider the middle degree cohomology

$$
\mathbb{H}=\lim _{m} H^{d}\left(X_{K_{m}} \times \overline{\mathbb{Q}}, V_{\lambda, K_{m}}\left(\overline{\mathbb{Q}}_{p}\right)\right)
$$

Note that any $\mathbb{Z}_{p}$-model $V_{\lambda}$ of the representation of $G$ defines a compatible system of integral étale sheaves $\left.V_{\lambda, K_{m}}\left(\overline{\mathbb{Z}}_{p}\right)\right)$, hence an integral structure $\mathbb{H}_{\mathbb{Z}_{p}}$ of $\mathbb{H}: \mathbb{H}=\mathbb{H}_{\mathbb{Z}_{p}} \otimes \mathbb{Q}_{p}$; note that $\mathbb{H}_{\mathbb{Z}_{p}}$ may have torsion. Let $\epsilon=(1, \ldots, 1 ; 0) \in X_{*}(T)$; we put $w=\langle\lambda+\rho, \epsilon\rangle$; it is the motivic weight of these cohomology groups.

For any prime $\ell$ not dividing $M$, let $d_{\ell, 1}=\operatorname{diag}\left(1_{n}, \ell \cdot 1_{n}\right)$ and for any $i=2 \ldots, n$, let $d_{\ell, i}=$ $\operatorname{diag}\left(1_{n+1-i}, \ell \cdot 1_{2 n-i}, \ell^{2} \cdot 1_{n+1-i}\right)$. Consider the groups $K_{m}^{\prime}=d_{\ell, i} K_{m} d_{\ell, i}^{-1}$ and $K_{m}^{\prime \prime}=d_{\ell, i}^{-1} K_{m} d_{\ell, i}$, we have an isomorphism $\pi\left(d_{\ell, i}\right): X_{K_{m}^{\prime}} \rightarrow X_{K_{m}^{\prime \prime}}$. We define an action of the double class $K_{m} d_{\ell, i} K_{m}$ on the pair $\left(X_{K_{m}}, V_{\lambda, K_{m}}\left(\overline{\mathbb{Q}}_{p}\right)\right)$ by using the two finite morphisms $\pi_{K_{m}^{\prime}, K_{m}}, \pi_{K_{m}^{\prime \prime}, K_{m}}$ and the morphism of sheaves

$$
\left(\pi\left(d_{\ell, i}\right), d_{\ell, i}^{*}\right): V_{\lambda, K_{m}^{\prime \prime}}\left(\overline{\mathbb{Q}}_{p}\right) \rightarrow V_{\lambda, K_{m}^{\prime}}\left(\overline{\mathbb{Q}}_{p}\right)
$$

where for $t \in T\left(\mathbb{Q}_{p}\right)$, we write $t^{*}=\nu(t) \cdot t^{-1} \in T\left(\mathbb{Q}_{p}\right)$; and we consider its action on $V_{\lambda}\left(\mathbb{Q}_{p}\right)$. Note that for any $\ell \neq p, d_{\ell, i}^{*}=d_{\ell, i}^{*}=1$ on $V_{\lambda}\left(\mathbb{Q}_{p}\right)$, in particular, it preserves any integral structure of $V_{\lambda}$. For $\ell=p$, We see that $d_{\ell, i}^{*}$ defines an isomorphism of $V_{\lambda}\left(\mathbb{Q}_{p}\right)$, that it preserves $V_{\lambda}^{\max }\left(\mathbb{Z}_{p}\right)=\operatorname{Ind}_{B^{-}}^{G} \lambda$, but has non trivial cokernel ; we won't make use of this integral structure in the sequel. We define for any prime $\ell$ not dividing $M$, and any $i=1, \ldots, n$ :

$$
\left.\left[K_{m} d_{\ell, i} K_{m}\right]=\pi_{K_{m}^{\prime}, K_{m}, *} \circ\left(\pi d_{\ell, i}, d_{\ell, i}^{*}\right)\right)^{*} \circ \pi_{K_{m}^{\prime \prime}, K_{m}}^{*} .
$$

These actions are compatible when $m$ varies and define $\mathbb{Q}_{p}$-linear endomorphisms on $\mathbb{H}$, denoted by $T_{\ell, i}$ for $\ell$ prime to $M p$, resp. by $U_{p, i}$ for $\ell=p$. These operators are the Hecke correspondences acting on $\mathbb{H}$. The space $\mathbb{H}$ carries a Galois action which commutes to the $T_{\ell, i}$ 's and $U_{p, i}$ 's. These actions preserve the integral structures.

It carries also a normal action of the torus $T\left(\mathbb{Z}_{p}\right)$ viewed as the quotient $B\left(\mathbb{Z}_{p}\right) / N\left(\mathbb{Z}_{p}\right)=$ $\varliminf_{\varlimsup} I_{m} / I_{m}^{+}$. This action is continuous. One can decompose $T\left(\mathbb{Z}_{p}\right)$ as $T_{f} \times T_{1}$ where $T_{f}$ is the torsion subgroup and $T_{1}$ is the pro- $p$-Sylow, kernel of the reduction modulo $p$. We fix a topological basis $\left(u_{1}, \ldots, u_{n}\right)$ of $T_{1}$ by fixing $u=1+p$ as topological basis of $1+p \mathbb{Z}_{p}$ and defining $u_{i}$ as the image of $u$ by the cocharacter $t \mapsto \operatorname{diag}\left(E_{i}(t), E_{n+1-i}\left(t^{-1}\right)\right)$ where $E_{i}(t)=\operatorname{diag}(1, \ldots, t, \ldots, 1)$ ( $t$ is the $i$-th component). By this choice, we identify the completed group algebra $\mathbb{Z}_{p}\left[\left[T_{1}\right]\right]$ to the $n$-variable Iwasawa algebra $\Lambda_{n}=\mathbb{Z}_{p}\left[\left[X_{1}, \ldots, X_{n}\right]\right]$ via $u_{i} \mapsto X_{i}+1$. $\mathbb{H}$ is therefore a $\Lambda_{n}\left[T_{f}\right]$-module. This action commutes to the $T_{\ell, i}$ and the $U_{p, i}$ 's ; moreover it preserves any integral structure given by a $\mathbb{Z}_{p}$-model $V_{\lambda}$ of the representation of $G$. We consider the largest subspace $H_{0}^{d}\left(X_{K_{m}} \times \overline{\mathbb{Q}}, V_{\lambda, K_{m}}\left(\overline{\mathbb{Q}}_{p}\right)\right)$, resp. $\mathbb{H}_{0}$, of $H^{d}\left(X_{K_{m}} \times \overline{\mathbb{Q}}, V_{\lambda, K_{m}}\left(\overline{\mathbb{Q}}_{p}\right)\right)$, resp. of $\mathbb{H}$, on which the eigenvalues of the operators $U_{p, i}$ are $p$-adic units ; it is the sum of all the generalized eigenspaces for such eigenvalues. We denote by $\mathbf{T}_{\lambda}^{M}\left(K_{m}\right)$ the $\mathbb{Z}_{p}$-algebra generated by the $T_{\ell, i}$ 's, the $U_{p, i}$ 's and the group action of $T\left(\mathbb{Z} / p^{m} \mathbb{Z}\right)$ on $H_{0}^{d}\left(X_{K_{m}} \times \overline{\mathbb{Q}}, V_{\lambda, K_{m}}\left(\overline{\mathbb{Q}}_{p}\right)\right)$; similarly, let $\mathbf{T}^{M}$ be the $\Lambda_{n}$-subalgebra of $\operatorname{End}_{\Lambda_{n}} \mathbb{H}_{0}$ generated by the $T_{\ell, i}$ 's, the $U_{p, i}$ 's and the action of $T_{f}$. Recall ([M04] Prop.6.4.1) that $\mathbb{H}_{0}$ is a finite $\Lambda_{n}$-module. Therefore, $\mathbf{T}^{M}$ is a finite $\Lambda_{n}$-algebra. Note however that it is not cuspidal, in general (this would mean that all specializations at cohomological weights would be cuspidal). It is conjectured that cuspidality holds for the localization of $\mathbf{T}^{M}$ at a "weakly non-Eisenstein" maximal ideal (assuming the existence of the $\widehat{G}$-valued $p$-adic Galois representation and its ordinarity at $p$, "weak non-Eisensteinness" means that the corresponding residual Galois representation is absolutely irreducible).

However, another obstacle in this definition (due to the presence of torsion in the cohomology groups) is that the exact control theorem has not been established for all cohomological weights, unless one makes some strong extra assumptions. This seems to prevent to show in full generality that $\mathbf{T}^{M}$ is torsion-free as a $\Lambda_{n}$-algebra. Let $\lambda^{\prime} \in X^{*}(T)$ be a dominant weight such that $\left.\lambda^{\prime}\right|_{T_{f}}=\left.\lambda\right|_{T_{f}}$, its restriction to $T_{1}$ defines by linearity and continuity an algebra homomorphism $\lambda^{\prime}: \Lambda_{n} \rightarrow \mathbb{Z}_{p}$; we denote by $P_{\lambda^{\prime}}$ the prime ideal of $\Lambda_{n}$ kernel of $\lambda^{\prime}$.

Let $C>0$ be a real number. The set of $C$-regular weights is the set of $\lambda^{\prime \prime}$ 's such that $\left\langle\lambda^{\prime}, \alpha\right\rangle>C$ for all simple roots $\alpha$ of $G$. 
Definition 4.1. We say that control theorem, resp. $\mathfrak{m}$-control theorem, holds for the weight $\lambda^{\prime}$ (congruent to $\lambda$ mod. $p-1$ ), if the surjective morphism $\mathbf{T}^{M} \otimes_{\Lambda_{n}, \lambda^{\prime}} \mathbb{Z}_{p} \rightarrow \mathbf{T}_{\lambda^{\prime}}^{M}\left(K_{1}\right)$, resp. $\mathbf{T}_{\mathfrak{m}}^{M} \otimes_{\Lambda_{n}, \lambda^{\prime}}$ $\mathbb{Z}_{p} \rightarrow \mathbf{T}_{\lambda^{\prime}}^{M}\left(K_{1}\right)_{\mathfrak{m}}$, has finite kernel.

If one assumes that $p-1>w$ (for the motivic weight $w$ ) and if the maximal ideal $\mathfrak{m}$ is "strongly non-Eisenstein" (in the sense of [MT02] Sect.9 : it means that the image of the residual representation contains the normalizer of the $\mathbb{F}_{p}$-points of the standard maximal torus $\widehat{T}$ ) then cuspidality and $\Lambda_{n}$-torsion-freeness hold for the $\mathfrak{m}$-localized Hecke algebra and $\mathfrak{m}$-control theorem do hold for all cohomological weights $\lambda^{\prime}$ congruent to $\lambda$ mod. $p-1$ (see Th.10 of [MT02]), otherwise, without assuming the existence of the Galois representations and without localization, there exists a constant $C$ depending on the data $(\lambda, p)$ such that control theorem holds for all $C$-regular weights (but it does not imply the torsion-freeness of the Hecke algebra, except for Siegel-Hilbert varieties of genus 2 , see [TU99], or for unitary groups in three variables, see [M04]).

In order to obtain a control theorem for all weights without localization, one needs to follow another approach via coherent cohomology, started by Hida [H02]. We identify the character group $X^{*}(T)$ to the index two sublattice of $\mathbb{Z}^{n+1}$ by sending the character

$$
\lambda: \operatorname{diag}\left(t_{1}, \ldots, t_{n}, \nu t_{n}^{-1}, \ldots, \nu t_{1}^{-1}\right) \mapsto t_{1}^{k_{1}} \ldots t_{n}^{k_{n}} \nu^{\left(c-k_{1}-\ldots-k_{n}\right) / 2}
$$

to $\left(k_{1}, \ldots, k_{n} ; c\right)$. Let $t=(1, \ldots, 1 ; 1)$; we fix the cohomological weight $k=\lambda+(n+1) t=$ $\left(k_{1}, \ldots, k_{n} ; k_{1}+\cdots+k_{n}\right)$ and the sheaf $\omega_{\text {cusp }}^{k}$ over $X_{K_{1}}$ (see [H02, Sect.2.3] and [Pi12, Sect.4]). These objects are now viewed on the rigid analytic site over $\mathbb{Q}_{p}$. One starts from a holomorphic cusp form $f \in H^{0}\left(X_{K_{1}}, \omega_{\text {cusp }}^{k}\right)$; instead of the whole tower of finite coverings $X_{K_{m}} \rightarrow X_{K_{1}}$, one forms the Igusa tower $T_{m}=\operatorname{Isom}_{X_{K_{1}}^{0}}\left(\mu_{p^{m}}^{n}, A\left[p^{m}\right]^{\circ}\right)$ over the ordinary locus $X_{K_{1}}^{0}$ of $X_{K_{1}}$ (here, $A\left[p^{m}\right]^{\circ}$ denotes the connected component of the $p^{m}$-torsion of the universal abelian variety over $\left.X_{K_{1}}^{0}\right) ; T_{m}$ can be viewed as the rigid tube of the "multiplicative type" connected component of the ordinary locus in the reduction modulo $p$ of $X_{K_{m}}$. Consider the $\mathbb{Q}_{p}$-vector space $\mathbb{H} \mathbb{C}=\lim _{m} H^{0}\left(T_{m}, \omega_{\text {cusp }}^{k}\right)$ ; one defines similarly action of the Hecke operators $T_{\ell, i}$ 's and $U_{p, i}$ 's $(i=1, \ldots, n)$; these are compatible with the transition morphisms of the Igusa tower ; the Galois group of the Igusa tower is $T\left(\mathbb{Z}_{p}\right)$ which therefore acts on $H^{0}\left(T_{m}, \omega_{\text {cusp }}^{k}\right)$ and $\mathbb{H} \mathbb{C}$. One defines the $\mathbb{Z}_{p}$-Hecke algebras $\mathbf{T}^{M}\left(T_{m}\right)$, resp. $\mathbf{T}^{M}\left(T_{\infty}\right)$ as generated by $T_{\ell, i}$ 's, $U_{p, i}$ 's and the image of $T\left(\mathbb{Z}_{p}\right)$ acting on $H^{0}\left(T_{m}, \omega^{k}\right)$, resp. $\mathbb{H} \mathbb{C}$. Similarly, one could define $\mathbf{T}^{M, \text { holcusp }}$ as the $\mathbb{Z}_{p}$-algebra acting on the ordinary part $\mathbb{H}_{0}^{\text {holcusp }}$ of $\mathbb{H}^{\text {holcusp }}=\lim _{m} H^{0}\left(X_{K_{m}}, \omega_{\text {cusp }}^{k}\right)$; by density of classical Siegel modular cusp forms in $p$-adic modu-

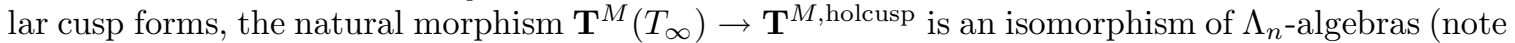
though that one has only surjections $\mathbf{T}^{M}\left(T_{m}\right) \rightarrow \mathbf{T}^{M \text {, holcusp }}\left(K_{m}\right)$ ). By Hodge-Tate decomposition (see Faltings-Chai Chapter VI, using BGG theory), $\mathbf{T}^{M \text {,holcusp }}$ is a quotient of $\mathbf{T}^{M}$ (it cannot be injective since the left-hand side contains non cuspidal families, but after localization at any weakly non-Eisenstein maximal ideal should be an isomorphism).

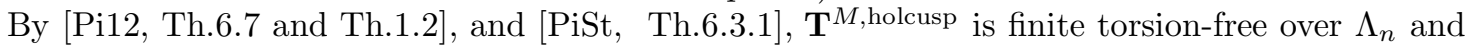
(cuspidal) Control Theorem holds without localization (and without assuming the existence of the Galois representations) ; in other words, for any dominant weight $\lambda^{\prime}$ such that $\left.\lambda^{\prime}\right|_{T_{f}}=\left.\lambda\right|_{T_{f}}$, the surjective morphism $\mathbf{T}^{M \text {,holcusp }} \otimes_{\Lambda_{n}, \lambda^{\prime}} \mathbb{Z}_{p} \rightarrow \mathbf{T}_{\lambda^{\prime}}^{M \text {,holcusp }}\left(K_{1}\right)$ has finite kernel.

Let $\mathbb{I}$ be the normal closure of an irreducible component of $\operatorname{Spec}\left(\mathbf{T}^{M \text {,holcusp }}\right)$. Let $\theta: \mathbf{T}^{M \text {,holcusp }} \rightarrow$ II be the corresponding Hida family. Assuming the existence of the Galois representations $\rho_{f}: G_{\mathbb{Q}} \rightarrow$ $\widehat{G}\left(\overline{\mathbb{Z}}_{p}\right)$ associated to the holomorphic cusp forms of level $K_{1}$ occurring in the family, let us construct the Galois representation $G_{\mathbb{Q}} \rightarrow \widehat{G}(\mathbb{I})$ associated to $\theta$. We say that a cusp eigenform $f^{\prime}$ of level $K_{1}$, weight $k^{\prime}=\lambda^{\prime}+(n+1) t$ and eigensystem $\theta_{f^{\prime}}$ occurs in the family if there exists a prime $P^{\prime}$ of $\mathbb{I}$ above $P_{\lambda^{\prime}}$ such that, via the control theorem, one has $\theta_{f^{\prime}}=\theta\left(\bmod P^{\prime}\right)$.

Definition 4.2. We say that the irreducible component $\mathbb{I}$ is weakly non-Eisenstein if for some (hence all) cusp eigenform $f^{\prime}$ occurring in the family, the residual representation composed with spin is absolutely irreducible.

Lemma 4.3. For any weakly non-Eisenstein irreducible component $\mathbb{I}$, there exists a continuous homomorphism $\rho_{\theta}: G_{\mathbb{Q}} \rightarrow \widehat{G}(\mathbb{I})$ such that for any dominant weight $\lambda^{\prime}$ such that $\left.\lambda^{\prime}\right|_{T_{f}}=\left.\lambda\right|_{T_{f}}$, and 
for any cusp eigenform $f^{\prime}$ of weight $k^{\prime}=\lambda^{\prime}+(n+1) t$ in the family, the representation $\rho_{f^{\prime}}$ is conjugate in $\widehat{G}\left(\overline{\mathbb{Q}}_{p}\right)$ to $\rho_{\theta}\left(\bmod P^{\prime}\right)$.

Proof. We give the proof only for $\mathbb{I}=\Lambda_{n}$. Let us consider the $\mathbb{Z}_{p}$-morphism Ad: $\widehat{G} \rightarrow \mathrm{SO}_{2 n+1}$; note that $\operatorname{Ker}(\mathrm{Ad})$ is the center $\widehat{Z} \cong \mathbb{G}_{m}$ of $\widehat{G}$. We first construct a representation $R: G_{\mathbb{Q}} \rightarrow \mathrm{SO}_{2 n+1}(\mathbb{I})$ and then lift it to $\widehat{G}(\mathbb{I})$. For any arithmetic prime $P^{\prime}$ of $\mathbb{I}$ above a dominant weight $\lambda^{\prime}$ such that $\left.\lambda^{\prime}\right|_{T_{f}}=\left.\lambda\right|_{T_{f}}$, let $f_{P^{\prime}}$ be a cusp eigenform occurring in the family with this weight ; by residual irreducibility of spin $\circ \rho_{f_{P^{\prime}}}$ and Carayol's theorem [Car94], we can assume that this representation is defined over $\mathbb{I} / P^{\prime}$; by injectivity of spin, it implies that $\rho_{P^{\prime}}=\rho_{f_{P^{\prime}}}$ takes values in $\widehat{G}\left(\mathbb{I} / P^{\prime}\right)$ : $\rho_{P^{\prime}}: G_{\mathbb{Q}} \rightarrow \widehat{G}\left(\mathbb{I} / P^{\prime}\right)$.

On the other hand, by residual irreducibility, it follows from Carayol's theorem [Car94] that Ad $\circ \bar{\rho}$ lifts to a continuous representation $R: G_{\mathbb{Q}} \rightarrow \mathrm{GL}_{2 n+1}(\mathbb{I})$. Since Ad $\circ \bar{\rho}$ is orthogonal, and each specialization modulo an arithmetic prime is orthogonal, we see that $R$ is orthogonal. Since $\mathbb{I}$ is profinite and by formal smoothness of the group $\widehat{G}$, there exists a continuous lift $\widetilde{R}: G_{\mathbb{Q}} \rightarrow \widehat{G}(\mathbb{I})$, which gives rise to a 2-cocyle $c_{\sigma, \tau} \in Z^{2}\left(G_{\mathbb{Q}}, \mathbb{I}^{\times}\right)$.

For any prime $P^{\prime}$, the existence of the representation $\rho_{P^{\prime}}$ implies that the image of $c_{\sigma, \tau}$ is a coboundary : $c_{\sigma, \tau}=b_{\sigma \tau} b_{\sigma}^{-1} b_{\tau}^{-1}$ over $\left(\mathbb{I} / P^{\prime}\right)^{\times}$. Take $\mathbb{I}=\Lambda_{n}$ and consider the Iwasawa ideal $\Omega_{n, k}$ generated by the $\left(1+T_{i}\right)^{p^{n}}-u^{k_{i} p^{n}},=1, \ldots, n$. The quotient ring $\Lambda_{n, \underline{k}}=\Lambda / \Omega_{n, \underline{k}}$ embeds in the product of cyclotomic rings $\Lambda\left(\zeta_{1}, \ldots, \zeta_{n}\right)=\Lambda_{n} / I\left(\zeta_{1}, \ldots, \zeta_{n}\right)$ where

$$
I\left(\zeta_{1}, \ldots, \zeta_{n}\right)=\left(1+T_{i}-u^{k_{i}} \zeta_{i}, \quad i=1 \ldots, n\right)
$$

where $\zeta_{i}$ is a $p^{n}$-th root of unity. The condition for a family $\left(b\left(\zeta_{1}, \ldots, \zeta_{n}\right)\right)_{\zeta_{1}, \ldots, \zeta_{n}}$ to be in the image of the embedding is that for any pair $\left(\zeta_{1}, \ldots, \zeta_{n}\right)$ and $\left(\zeta_{1}^{\prime}, \ldots, \zeta_{n}^{\prime}\right)$ the components $b\left(\zeta_{1}, \ldots, \zeta_{n}\right)$ and $b\left(\zeta_{1}^{\prime}, \ldots, \zeta_{n}^{\prime}\right)$ are congruent modulo $I_{(}\left(\zeta_{1}, \ldots, \zeta_{n}\right)+I\left(\zeta_{1}^{\prime}, \ldots, \zeta_{n}^{\prime}\right)$. Let us check it is the case for the family of 1-cochains $\left(b\left(\zeta_{1}, \ldots, \zeta_{n}\right)_{\sigma}\right)$ associated to the specialization $c_{\sigma, \tau}^{n, \frac{k}{\tau}}$ of $c_{\sigma, \tau}$ over $\left(\Lambda_{n, k}\right)^{\times}$. Indeed, let us compare the reductions modulo $I\left(\zeta_{1}, \ldots, \zeta_{n}\right)+I\left(\zeta_{1}^{\prime}, \ldots, \zeta_{n}^{\prime}\right)$ of the representations spin $\circ \widetilde{R}(\sigma) b\left(\zeta_{1}, \ldots, \zeta_{n}\right)_{\sigma}$ and spin $\circ \widetilde{R}(\sigma) b\left(\zeta_{1}^{\prime}, \ldots, \zeta_{n}^{\prime}\right)_{\sigma}$; they have the same characteristic polynomial and they both lift the irreducible representation spin $\circ \bar{\rho}$. By Carayol's theorem [Car94], they are conjugate, so that $b\left(\zeta_{1}, \ldots, \zeta_{n}\right)_{\sigma}=b\left(\zeta_{1}^{\prime}, \ldots, \zeta_{n}^{\prime}\right)_{\sigma}$ in $\Lambda_{n} / I\left(\zeta_{1}, \ldots, \zeta_{n}\right)+I\left(\zeta_{1}^{\prime}, \ldots, \zeta_{n}^{\prime}\right)$.

From this, we conclude that there exists a 1 -cochain $b_{\sigma}^{n, \underline{k}}$ with values in $\left(\Lambda_{n, \underline{k}}\right)^{\times}$such that $c_{\sigma, \underline{\tau}}^{n, \underline{k}}=$ $b_{\sigma \tau}^{n, \underline{k}} b_{\sigma}^{n, \underline{k}-1} b_{\tau}^{n, \underline{k}-1}$. This construction is compatible when $n$ grows and gives rise to a 1-cochain $b_{\sigma}$ with values in $\Lambda_{n}^{\times}$as desired. The resulting map $\sigma \mapsto \widetilde{R}(\sigma) b_{\sigma}$ is the desired representation $G_{\mathbb{Q}} \rightarrow$ $\widehat{G}\left(\Lambda_{n}\right)$.

Moreover $\rho_{\theta}$ is ordinary at $p$. More precisely, recall that $\epsilon$ denotes the $p$-adic cyclotomic character ; we define for any $\sigma \in \operatorname{Gal}(\overline{\mathbb{Q}} / \mathbb{Q})$ the cyclotomic logarithm $\ell(\sigma)=\frac{\log _{p}(\epsilon(\sigma))}{\log _{p}(1+p)}$ (for the $p$-adic cyclotomic character $\epsilon)$. Let $t_{i}=1+X_{i}(i=1, \ldots, n)$, then

Lemma 4.4. Assume $p-1>w$. The representation $\rho_{\theta}$ is ordinary : there exists $\widehat{g} \in \widehat{G}(\mathbb{I})$ such that $\rho_{\theta}\left(D_{p}\right) \subset \widehat{g} \widehat{B}(\mathbb{I}) \widehat{g}^{-1}$ and for any $\sigma \in I_{p}$,

$$
\widehat{g}^{-1} \rho_{\theta}(\sigma) \widehat{g} \equiv\left[\epsilon(\sigma)^{-1} t_{1}^{\ell(\sigma)}, \epsilon(\sigma)^{-2} t_{2}^{\ell(\sigma)} \ldots, \epsilon(\sigma)^{-n} t_{n}^{\ell(\sigma)} ; \epsilon(\sigma)^{-d}\right] \quad(\bmod U(\mathbb{I}))
$$

where $d=\frac{n(n+1)}{2}$.

Proof. Since all Borel subgroups are conjugated in $\widehat{G}$, it is enough to apply the spin representation and to find a $D_{p}$-stable flag in $\mathbb{I}^{2^{n}}$ with action of $I_{p}$ on the graded pieces given by the mutually distinct characters $\prod_{i \in I} \epsilon(\sigma)^{-i} t_{i}^{\ell(\sigma)}$ for all subset $I^{\prime}$ 's of $\{1, \ldots, n\}$. Over $\mathbb{J}=\prod_{P^{\prime}} \mathcal{O}_{P^{\prime}}$ as in the previous proof, we have such a flag (in $\mathbb{J}^{2^{n}}$ ). If we assume $p-1>w$ so that all the characters above take values in $\mathbb{I}$ and are mutually distinct modulo $p$, we see that the intersection of this flag with $\mathbb{I}^{2^{n}}$ induces a flag as desired. 
4.3. $\Lambda_{n}$-fullness. In the sequel, we consider an irreducible component $\mathbb{I}$ of $\mathbf{T}^{M}$ such that $\mathbb{I}=\Lambda_{n}=$ $\mathbb{Z}_{p}\left[\left[X_{1}, \ldots X_{n}\right]\right]$ (with $p>2$ ). Let $u=1+p$. Let $\mathbb{Q}_{p}^{\text {cyc }}$ be the $p$-power-cyclotomic extension of $\mathbb{Q}_{p}$. For any weight $\underline{k}=\left(k_{1}, \ldots, k_{n}\right)$ dominant for $(G, B, T)$, and cohomological, we denote by $P_{k}$ the point of Spec $\Lambda_{n}$ given by $P_{k}=\left(1+X_{1}-u^{k_{1}}, \ldots, 1+X_{n}-u^{k_{n}}\right)$. the point

We define for any $\sigma \in \operatorname{Gal}(\overline{\mathbb{Q}} / \mathbb{Q})$ the cyclotomic logarithm $\ell(\sigma)=\frac{\log _{p}(\epsilon(\sigma))}{\log _{p}(1+p)}$ (for the $p$-adic cyclotomic character $\epsilon)$. We put, as above, $t_{i}=1+X_{i}(i=1, \ldots, n)$, and we assume that the semi-simplification of $\rho$ restricted to the $p$-inertia group $I_{p}$ is

$$
\left[\epsilon(\sigma)^{-1} t_{1}^{\ell(\sigma)}, \epsilon(\sigma)^{-2} t_{2}^{\ell(\sigma)} \ldots, \epsilon(\sigma)^{-n} t_{n}^{\ell(\sigma)} ; \epsilon(\sigma)^{-d}\left(t_{1} \cdot \ldots \cdot t_{n}\right)^{\ell(\sigma)}\right],
$$

where $d=\frac{n(n+1)}{2}$. This is conjecturally the case if $\rho$ is associated to an $n$-variable $p$-adic Hida family of Siegel forms of genus $n$, passing by a form $f$ of level prime to $p$ and weight $\underline{k^{0}}=\left(k_{1}^{0}, \ldots, k_{n}^{0}\right)$, with $k_{1}^{0} \geq \ldots \geq k_{n}^{0} \geq n+1$. It is actually proven if $n=2$.

We consider the Galois representation $\rho=\rho_{\theta}: \operatorname{Gal}(\overline{\mathbb{Q}} / \mathbb{Q}) \rightarrow \operatorname{GSpin}_{2 n+1}\left(\Lambda_{n}\right)$ associated to the family $\theta: \mathbf{T}^{M \text {,holcusp }} \rightarrow \mathbb{I}=\Lambda_{n}$; the representation is $\rho$ ordinary at $p$; After conjugation, we can and will assume in the sequel that $\rho\left(D_{p}\right) \subset \widehat{B}\left(\Lambda_{n}\right)$. For any root $\alpha$ of $(\widehat{G}, \widehat{T})$, we denote by $U_{\alpha}$ the one-dimensional Lie subgroup of $\operatorname{Spin}_{2 n+1}$, by $u_{\alpha}: \mathbf{G}_{a} \rightarrow U_{\alpha}$ the corresponding one-parameter subgroup and by $\mathfrak{u}_{\alpha}=\operatorname{Lie}\left(U_{\alpha}\right) \subset \mathfrak{s p i n}_{2 n+1}$ the corresponding Lie subalgebra (say over $\mathbb{Z}_{p}$ or any base change thereof). Because of these notations, it will be convenient to write $U$ instead of $\widehat{N}$ for the unipotent radical of the standard Borel subgroup $\widehat{B}=\widehat{T} \widehat{N}$. We have the following key lemma

Lemma 4.5. Let $\rho: \operatorname{Gal}(\overline{\mathbb{Q}} / \mathbb{Q}) \rightarrow \operatorname{GSpin}_{2 n+1}\left(\Lambda_{n}\right)$ be the Galois representation associated to a

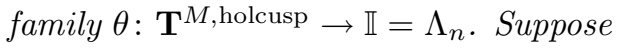

(1) there is a cohomological weight $k^{0}$ for which the specialization $\rho_{P_{k} 0}=\rho_{f}$ has $\mathbb{Z}_{p}$-full image; in other words, $\operatorname{Im}\left(\rho_{P_{k^{0}}}\right)$ contains an open subgroup of $\operatorname{Spin}_{2 n+1}\left(\mathbb{Z}_{p}\right)$,

(2) strong regularity, (compare to 3.5) $\operatorname{Im}(\rho)$ contains an element $\delta \in \widehat{T}\left(\Lambda_{n}\right)$ such that for any two roots $\alpha \neq \alpha^{\prime}$ of $\mathrm{SO}_{2 n+1}, \alpha(\operatorname{Ad}(\delta)) \not \equiv \alpha^{\prime}(\operatorname{Ad}(\delta))\left(\bmod \mathfrak{m}_{\Lambda_{n}}\right)$,

Then for any root $\alpha$ of $(\widehat{G}, \widehat{T}), \operatorname{Im} \rho \cap U_{\alpha}\left(\Lambda_{n}\right) \neq 0$

\section{Remarks :}

1) Note that the roots of $\left(\widehat{G}=\mathrm{GSpin}_{2 n+1}, \widehat{T}\right)$ are the same as those of $\left(\mathrm{SO}_{2 n+1}, T_{\mathrm{SO}_{2 n+1}}\right)$.

2) If instead of Assumption 2 we only assume that there exists an element $\delta \in \widehat{B}\left(\Lambda_{n}\right)$ such that its projection $\delta_{1} \in \widehat{T}\left(\Lambda_{n}\right)$ is strongly regular mod. $\mathfrak{m}_{\Lambda_{n}}$, by which we mean that for any two roots $\alpha \neq \alpha^{\prime}$ of $\mathrm{SO}_{2 n+1}, \alpha\left(\operatorname{Ad}\left(\delta_{1}\right)\right) \not \equiv \alpha^{\prime}\left(\operatorname{Ad}\left(\delta_{1}\right)\right)\left(\bmod \mathfrak{m}_{\Lambda_{n}}\right)$, then, we can modify it by conjugations by elements $u_{\alpha}(x)$ for positive roots $\alpha$ roots and suitable elements $x$ so that it belongs to $\widehat{T}\left(\Lambda_{n}\right)$ and it satisfies the exact statement of Assumption 2.

3) If we assume furthermore that $p-1>2 w$ and $\underline{k}^{0}=\left(k_{1}^{0}, \ldots, k_{n}^{0}\right)$ is such that for any $i \neq j$, $k_{i}^{0}-i \not \equiv \pm\left(k_{j}^{0}-j\right)(\bmod p-1)$, we see by Lemma 4.4 above that if we choose $\sigma \in I_{p}$ such that $\kappa(\sigma)=\omega(\sigma)$ is of exact order $p-1$, we can take $\delta=\rho(\sigma)$.

Proof. Since the argument is the same for positive and negative roots, we restrict ourselves to the "upper triangular" unipotent radical $U$ (corresponding to positive roots $\alpha$ ) of $\operatorname{Spin}(2 n+1)$. Let $K=\operatorname{Im} \rho \subset \widehat{G}\left(\Lambda_{n}\right)$. We write $P=P_{k^{0}}$ for the arithmetic point such that the specialization $\rho_{P}$ of the representation $\rho$ is $\mathbb{Z}_{p}$-full. Fix a positive root $\alpha$ and define

$$
\begin{aligned}
& \Gamma_{U_{\alpha}}\left(P^{j}\right)=\left\{x \in \operatorname{Spin}_{2 n+1}\left(\Lambda_{n}\right) \mid x \quad \bmod P^{j} \in U_{\alpha}\left(\Lambda_{n} / P^{j}\right)\right\} \\
& \Gamma_{B_{\alpha}}\left(P^{j}\right)=\left\{x \in \operatorname{Spin}_{2 n+1}\left(\Lambda_{n}\right) \mid x \quad \bmod P^{j} \in \widehat{T}\left(\Lambda_{n} / P^{j}\right) U_{\alpha}\left(\Lambda_{n} / P^{j}\right)\right\}
\end{aligned}
$$

and

$$
\begin{aligned}
K_{U_{\alpha}}\left(P^{j}\right) & =K \cap \Gamma_{U_{\alpha}}\left(P^{j}\right) \\
K_{B_{\alpha}}\left(P^{j}\right) & =K \cap \Gamma_{B_{\alpha}}\left(P^{j}\right)
\end{aligned}
$$

Since $U\left(\Lambda_{n}\right)$ and $\Gamma_{\Lambda_{n}}(P)$ are $p$-profinite, the groups $\Gamma_{U_{\alpha}}\left(P^{j}\right)$ and $K_{U_{\alpha}}\left(P^{j}\right)$ for all $j \geq 1$ are also $p$-profinite. Recall

$$
\Gamma_{\Lambda_{n}}\left(P^{i}\right)=\left\{x \in \operatorname{Spin}_{2 n+1}\left(\Lambda_{n}\right) \mid\left(x \bmod P^{i}\right)=1\right\}
$$


is the kernel of the reduction morphism $\pi_{j}: \operatorname{Spin}_{2 n+1}\left(\Lambda_{n}\right) \rightarrow \operatorname{Spin}_{2 n+1}\left(\Lambda_{n} / P^{j}\right)$. Recall the embedding $\iota_{\alpha}: \mathrm{SL}(2) \rightarrow \operatorname{Spin}_{2 n+1}$ associated to the root $\alpha$ (i.e., the upper (resp. lower) unipotent subgroup $U_{\mathrm{SL}(2)}^{+}\left(\right.$resp. $\left.U_{\mathrm{SL}(2)}^{-}\right)$of $\mathrm{SL}(2)$ is sent to $U_{\alpha}$ (resp. $U_{-\alpha}$ ) by $\iota_{\alpha}$ ). Thus, we see

$$
\Gamma_{U_{\alpha}}\left(P^{j}\right)=\Gamma_{\Lambda_{n}}\left(P^{j}\right) \iota_{\alpha}\left(U_{\mathrm{SL}(2)}\left(\Lambda_{n}\right)\right) .
$$

Note that

From this, we have

$$
\left[\left(\begin{array}{cc}
a & b \\
c & -a
\end{array}\right),\left(\begin{array}{cc}
e & f \\
g & -e
\end{array}\right)\right]=\left(\begin{array}{cc}
b g-c f & 2(a f-b e) \\
2(c e-a g) & c f-b g
\end{array}\right) .
$$

Lemma 4.6. If $X, Y \in \mathfrak{s l}_{2}\left(\Lambda_{n}\right) \cap\left(\begin{array}{cc}P^{j} & P^{k} \\ P^{i} & P^{j}\end{array}\right)$ with $i \geq j \geq k,[X, Y] \in\left(\begin{array}{ll}P^{i+k} & P^{j+k} \\ P^{i+j} & P^{i+k}\end{array}\right)$.

This tells us, for the topological commutator subgroup $D \Gamma_{U_{\alpha}}\left(P^{j}\right):=\left(\Gamma_{U_{\alpha}}\left(P^{j}\right), \Gamma_{U_{\alpha}}\left(P^{j}\right)\right)$ that

$$
D \Gamma_{U_{\alpha}}\left(P^{j}\right) \subset \Gamma_{B_{\alpha}}\left(P^{2 j}\right) \cap \Gamma_{U_{\alpha}}\left(P^{j}\right) .
$$

Using now the $\mathbb{Z}_{p}$-regularity assumption (in Section 3.5), we consider an element an element $\delta \in$ $\widehat{T}\left(\Lambda_{n}\right) \in K$, with distinct root values modulo $\mathfrak{m}_{\Lambda_{n}}$. Replacing $\delta$ by $\lim _{m \rightarrow \infty} \delta^{p^{m}}$, we may assume that $\delta \in \widehat{T}\left(\mathbb{Z}_{p}\right)$ has finite order $a$. The order $a$ is prime to $p$ (indeed it is a factor of $p-1$ ). Note that $\delta$ normalizes $K_{U_{\alpha}}\left(P^{j}\right)$ and $\Gamma_{B_{\alpha}}\left(P^{j}\right)$. Since $H$ is $p$-profinite, $x \in H$ has unique $a$-th root inside $H$. We define

$$
\Delta_{\alpha}(x)=\sqrt[a]{x \cdot\left(\delta x \delta^{-1}\right)^{\alpha(\delta)^{-1}} \cdot\left(\delta^{2} x \delta^{-2}\right)^{\alpha(\delta)^{-2}} \cdots\left(\delta^{a-1} x \delta^{1-a}\right)^{\alpha(\delta)^{1-a}}} \in H .
$$

Lemma 4.7. If $u \in \Gamma_{U_{\alpha}}\left(P^{j}\right)(j \geq 1)$, then $\Delta_{\alpha}^{2}(u) \in \Gamma_{U_{\alpha}}\left(P^{2 j}\right)$ and $\pi_{j}\left(\Delta_{\alpha}(u)\right)=\pi_{j}(u)$.

Proof. If $u \in \Gamma_{U_{\alpha}}\left(P^{j}\right)$, we have $\pi_{j}\left(\Delta_{\alpha}(u)\right)=\pi_{j}(u)$ as $\Delta_{\alpha}$ is the identity map on $U_{\alpha}\left(\Lambda_{n} / P^{j}\right)$. Let $D \Gamma_{U_{\alpha}}\left(P^{j}\right)$ be the topological commutator subgroup of $\Gamma_{U_{\alpha}}\left(P^{j}\right)$. Since $\Delta_{\alpha}$ induces the pro-

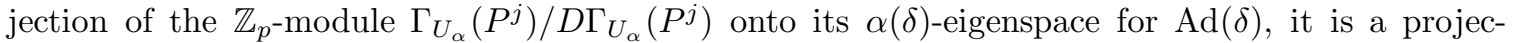
tion onto $U_{\alpha}\left(\Lambda_{n}\right) D \Gamma_{U_{\alpha}}\left(P^{j}\right) / D \Gamma_{U_{\alpha}}\left(P^{j}\right)$. The fact that this is exactly the $\alpha(\delta)$-eigenspace comes from the Iwahori decomposition of $\Gamma_{U_{\alpha}}\left(P^{j}\right)$ which shows it is generated by the $U_{\beta}\left(P^{j} \Lambda_{n}\right)$ for all roots $\beta \neq \alpha$ and by $U_{\alpha}\left(\Lambda_{n}\right)$, hence a similar direct sum decomposition holds in the abelianization $\Gamma_{U_{\alpha}}\left(P^{j}\right) / D \Gamma_{U_{\alpha}}\left(P^{j}\right)$.

By (4.3) $D \Gamma_{U_{\alpha}}\left(P^{j}\right) \subset \Gamma_{B_{\alpha}}\left(P^{2 j}\right) \cap \Gamma_{U_{\alpha}}\left(P^{j}\right)$. Since the $\alpha(\delta)$-eigenspace of $\Gamma_{U_{\alpha}}\left(P^{j}\right) / D \Gamma_{U_{\alpha}}\left(P^{j}\right)$ is inside $\Gamma_{B_{\alpha}}\left(P^{2 j}\right), \Delta_{\alpha}$ projects $u \Gamma_{U_{\alpha}}\left(P^{j}\right)$ to

$$
\bar{\Delta}_{\alpha}(u) \in\left(\Gamma_{B_{\alpha}}\left(P^{2 j}\right) \cap \Gamma_{U_{\alpha}}\left(P^{j}\right)\right) / D \Gamma_{U_{\alpha}}\left(P^{j}\right) .
$$

In particular, $\Delta_{\alpha}(u) \in \Gamma_{B_{\alpha}}\left(P^{2 j}\right) \cap \Gamma_{U_{\alpha}}\left(P^{j}\right)$. Again apply $\Delta_{\alpha}$. Since $\Gamma_{B_{\alpha}}\left(P^{2 j}\right) / \Gamma_{\Lambda_{n}}\left(P^{2 j}\right)$ is sent to to $\Gamma_{U_{\alpha}}\left(P^{2 j}\right) / \Gamma_{\Lambda_{n}}\left(P^{2 j}\right)$ by $\Delta_{\alpha}$, we get $\Delta_{\alpha}^{2}(u) \in \Gamma_{U_{\alpha}}\left(P^{2 j}\right)$ as desired.

We can now prove the key Lemma 4.5. Pick $0 \neq \bar{u}_{\alpha} \in U_{\alpha}(A / P) \cap \operatorname{Im}\left(\rho_{P}\right)$. By $\mathbb{Z}_{p}$-fullness of $\rho_{P}$, $\bar{u}_{\alpha}$ exists. Since the reduction map $\operatorname{Im}(\rho) \rightarrow \operatorname{Im}\left(\rho_{1}\right)$ is surjective, we have $u_{\alpha} \in \operatorname{Im}(\rho)$ such that $u_{\alpha}$ $\bmod P=\bar{u}_{\alpha}$. Take $v_{1} \in U_{\alpha}\left(\Lambda_{n}\right)$ such that $v_{1} \bmod P=\bar{u}_{\alpha}$. Such a $v_{1}$ exists as the reduction map $U_{\alpha}\left(\Lambda_{n}\right) \rightarrow U_{\alpha}\left(\Lambda_{n} / P\right)$ is onto. Then $u_{\alpha} v_{1}^{-1} \in \Gamma_{\Lambda_{n}}(P)$, we find that $u_{\alpha} \in K_{U_{\alpha}}(P)$.

By the compactness of $K_{U_{\alpha}}(P)$, by Lemma 4.7, starting with $u_{\alpha}$ as above, $\lim _{m \rightarrow \infty} \Delta_{\alpha}^{m}\left(u_{\alpha}\right)$ converges $P$-adically to $\Delta_{\alpha}^{\infty}\left(u_{\alpha}\right) \in U_{\alpha}\left(\Lambda_{n}\right) \cap \operatorname{Im}(\rho)$ with $\Delta_{\alpha}^{\infty}\left(u_{\alpha}\right) \Gamma_{\Lambda_{n}}^{\operatorname{Spin}(2 n+1)}(P)=u_{\alpha} \Gamma_{\Lambda_{n}}^{\operatorname{Spin}(2 n+1)}(P)$ (i.e., $\bar{u}_{\alpha}=\left(\Delta_{\alpha}^{\infty}\left(u_{\alpha}\right) \bmod P\right)$ ). Thus $\Delta_{\alpha}^{\infty}\left(u_{\alpha}\right)$ is a non-trivial unipotent element in $U_{\alpha}\left(\Lambda_{n}\right) \cap$ $\operatorname{Im}(\rho)$.

From this lemma we can deduce the following main theorem (we still assume that $\rho\left(D_{p}\right) \subset \widehat{B}\left(\Lambda_{n}\right)$ ).

Theorem 4.8. Let $\rho: \operatorname{Gal}(\overline{\mathbb{Q}} / \mathbb{Q}) \rightarrow \operatorname{GSpin}_{2 n+1}\left(\Lambda_{n}\right)$ be a continuous representation. Suppose

(1) there is a cohomological weight $k^{0}$ for which the specialization $\rho_{P_{k^{0}}}=\rho_{f}$ has $\mathbb{Z}_{p}$-full image ; in other words, $\operatorname{Im}\left(\rho_{P_{k^{0}}}\right)$ contains an open subgroup of $\operatorname{Spin}_{2 n+1}\left(\mathbb{Z}_{p}\right)$,

(2) $\operatorname{Im}(\rho)$ contains an element $\delta \in \widehat{B}\left(\Lambda_{n}\right)$, whose projection $\delta_{1} \in \widehat{T}\left(\Lambda_{n}\right)$ is $\mathfrak{s p i n}_{2 n+1}$-regular mod. $\mathfrak{m}_{\Lambda_{n}}$, that means that for any two roots $\alpha \neq \alpha^{\prime}$ of $\mathrm{SO}_{2 n+1}, \alpha\left(\operatorname{Ad}\left(\delta_{1}\right)\right) \not \equiv \alpha^{\prime}\left(\operatorname{Ad}\left(\delta_{1}\right)\right)$ $\left(\bmod \mathfrak{m}_{\Lambda_{n}}\right)$,

Then $\operatorname{Im} \rho$ contains a principal congruence subgroup of $\operatorname{Spin}_{2 n+1}\left(\Lambda_{n}\right)$ 
Definition 4.9. The largest ideal of $\Lambda_{n}$ such that $\Gamma_{\Lambda_{n}}\left(\mathfrak{l}_{\theta}\right) \subset \operatorname{Im} \rho_{\theta}$ is called the Galois level of $\theta$, and is denoted by $\mathfrak{l}_{\theta}$.

There are two proofs. It can either be deduced from Pink's theory using Lemma 3.6, as the only missing point was the non-triviality of $U_{\alpha}\left(\Lambda_{n}\right) \cap \operatorname{Im}(\rho)$ for all $\alpha$ 's (which follows from Lemma 4.5), or it can be proven using Lie algebras as follows.

Let $H=\operatorname{Im} \rho \cap \Gamma_{\Lambda_{n}}(p)$. We have seen that for any root $\alpha, \operatorname{Im} \rho \cap U_{\alpha}\left(\Lambda_{n}\right) \neq\{1\}$; therefore, by taking possibly a $p$-th power of th non trivial element in this intersection, we can assume that for any root $\alpha, H \cap U_{\alpha}\left(\Lambda_{n}\right) \neq\{1\}$. The series defining Log still provides a bijection between $H$ and the set $\log H$ which is contained in $p \cdot \mathfrak{s p i n}_{2 n+1}\left(\Lambda_{n}\right)$, but may not be stable by addition; however, the Log map induces group isomorphisms from $H \cap U_{\alpha}\left(\Lambda_{n}\right)$ to the (possibly infinite dimensional) $\mathbb{Z}_{p}$-Lie algebras $\log (H) \cap \mathfrak{u}_{\alpha}(\Lambda)$, which are therefore non-trivial $\mathbb{Z}_{p}$-modules for all roots $\alpha$. Recall that the $\mathbb{Q}_{p} \cdot \log (H)$ is a $\mathbb{Q}_{p}$-Lie algebra, denoted $\log _{\mathbb{Q}_{p}} H$ below.

Using the adjoint action of $\delta$, we have a decomposition into root spaces

$$
(*) \quad \log _{\mathbb{Q}_{p}} H=\left(\log _{\mathbb{Q}_{p}} H\right)_{0} \oplus \bigoplus_{\alpha \in \Phi}\left(\log _{\mathbb{Q}_{p}} H\right)_{\alpha}
$$

compatible with the root decomposition

$$
\mathfrak{s p i n}_{2 n+1}=\operatorname{Lie}(\widehat{T}) \oplus \bigoplus_{\alpha} \mathfrak{u}_{\alpha}
$$

which is valid over $\Lambda_{n}$, hence also over $\Lambda_{n}\left[\frac{1}{p}\right]$

Let $\left[t_{1}, \ldots, t_{n}\right]=\prod_{i}\left[e_{i}-\frac{1}{2} f\right]\left(t_{i}\right)$ denote the parametrization of (the semisimple part of ) the standard torus $\mathcal{T}^{\prime}=\widehat{T}$ of $\widehat{G}=\operatorname{GSpin}_{2 n+1}$ via $\widehat{T}=\mathbb{G}_{m} \otimes X^{*}(T)$, where $e_{i}:\left(x_{1}, \ldots, x_{n}, \nu x_{n}^{-1}, \ldots, \nu x_{1}^{-1}\right) \mapsto$ $x_{i}$ and $f:\left(x_{1}, \ldots, x_{n}, \nu x_{n}^{-1}, \ldots, \nu x_{1}^{-1}\right) \mapsto \nu$. For any $\sigma \in I_{p}$, we put

$$
t(\sigma)=\left[\left(1+T_{1}\right)^{\ell(\sigma)}, \ldots,\left(1+T_{n}\right)^{\ell(\sigma)}\right]
$$

We know that for any $\sigma \in I_{p}$,

$$
\rho^{\prime}(\sigma) \equiv t(\sigma) \quad\left(\bmod U\left(\Lambda_{n}\right)\right)
$$

The simple roots of $\widehat{G}$ (for the standard "upper triangular" Borel) are $\alpha_{i}:\left[t_{1}, \ldots, t_{n}\right] \mapsto t_{i} / t_{i+1}$ $(i=1, \ldots, n-1)$ and $\alpha_{n}:\left[t_{1}, \ldots, t_{n}\right] \mapsto t_{n}$. The positive roots are then $t_{i} / t_{j}$ for $1 \leq i<j \leq n, t_{i}, i=$ $1, \ldots, n$, and , $t_{i} t_{j}$ for $1 \leq i<j \leq n$; they can be written respectively as $\alpha_{i}+\ldots+\alpha_{j-1}, \alpha_{i}+\ldots+\alpha_{n}$, and $\alpha_{i}+\ldots+\alpha_{j-1}+2\left(\alpha_{j}+\ldots+\alpha_{n}\right)$. Conjugation by $\rho(\sigma)$ acts on $\log _{\mathbb{Q}_{p}} H_{\alpha}=\log _{\mathbb{Q}_{p}} H \cap \mathfrak{u}_{\alpha}\left(\Lambda_{n}\right)$ by $\alpha(t(\sigma))$; one can choose $\sigma \in I_{p}$ such that $\ell(\sigma)=1$. We find that we can let $\frac{1+X_{i}}{1+X_{j}}$, resp. $1+X_{i}$, resp. $\left(1+X_{i}\right)\left(1+X_{j}\right)$ act on $\left(\log _{\mathbb{Q}_{p}} H\right)_{t_{i} / t_{j}}$ resp. $\log _{\mathbb{Q}_{p}} H_{t_{i}}$, resp. $\left(\log _{\mathbb{Q}_{p}} H\right)_{t_{i} t_{j}}$. We have an action of $1+X_{n}$ on the non-zero subgroup $\left(\log _{\mathbb{Q}_{p}} H\right)_{\alpha_{n}}$ and of $\frac{1+X_{n-1}}{1+X_{n}}$ on the non-zero subgroup $\left(\log _{\mathbb{Q}_{p}} H\right)_{\alpha_{n-1}}$; by forming the Poisson bracket of these two submodules, we find a non-zero modules of $\left(\log _{\mathbb{Q}_{p}} H\right)_{\alpha_{n-1}+\alpha_{n}}$ on which both $\left(1+X_{n}\right)$ and $\frac{1+X_{n-1}}{1+X_{n}}$ act ; therefore $\left(1+X_{n}\right)$ and $1+X_{n-1}$ both act on this non zero subgroup of $\left(\log _{\mathbb{Q}_{p}} H\right)_{\alpha_{n-1}+\alpha_{n}}$. By repeating this procedure, we find, for $\alpha=\alpha_{1}+\ldots+\alpha_{n}$, a non-zero submodule of $\left(\log _{\mathbb{Q}_{p}} H\right)_{\alpha}$ on which all $1+X_{i}$ 's act. This is a non zero $\Lambda_{n}$-submodule of $U_{\alpha}\left(\Lambda_{n}\right)$, contained in $(\log H)_{\alpha}$. We then use successive Poisson brackets with $\left(\log _{\mathbb{Q}_{p}} H\right)_{-\alpha_{1}}, \ldots,\left(\log _{\mathbb{Q}_{p}} H\right)_{-\alpha_{j}}$ to construct inside all $\left(\log _{\mathbb{Q}_{p}} H\right)_{\alpha_{i}}$ 's a non-trivial $\Lambda_{n}$-submodule of $\mathfrak{u}_{\alpha_{i}}\left(\Lambda_{n}\right)$. Once we have non-zero $\Lambda_{n}$-submodules in $\left(\log _{\mathbb{Q}_{p}} H\right)_{\alpha_{i}}$ for all the simple roots, one can construct non-trivial $\Lambda_{n}$-submodules in $\left(\log _{\mathbb{Q}_{p}} H\right)_{\alpha}$ for all the roots $\alpha$. After multiplying by a power of $p$, we obtain non-trivial $\Lambda_{n}$-submodules in $\left(\log _{\mathbb{Q}_{p}} H\right)_{\alpha}$ for all roots. Taking the exponential, we see that there exists a non zero ideal $\mathfrak{l}^{\prime}$ of $\Lambda_{n}$ such that $U_{\alpha}\left(\mathfrak{l}^{\prime}\right) \subset \operatorname{Im} \rho$. Since these subgroups generate a group containing a principal congruence subgroup of $\operatorname{Spin}_{2 n+1}\left(\Lambda_{n}\right)$, we see that there exists a non zero ideal $\mathfrak{l}$ of $\Lambda_{n}$ such that $\Gamma_{\Lambda_{n}}(\mathfrak{l}) \subset \operatorname{Im} \rho$ as desired.

We give a lemma which follows from the proof above. For any simple root $\alpha_{i}$ of $\mathfrak{s p i n}_{2 n+1}$, let $\overline{\mathfrak{u}}_{\alpha_{i}}=\mathfrak{u}_{\alpha_{i}}\left(\Lambda_{n}\left[\frac{1}{p}\right]\right) \cap \operatorname{Lie}_{\mathbb{Q}_{p}} \operatorname{Im} \rho_{\theta}$. For any characteristic zero quotient $A$ of $\Lambda_{n}$, let $\rho_{A}$ be the 
specialization of $\rho_{\theta}$ to $A$ and let $\overline{\mathfrak{u}}_{\alpha_{i}}^{A}$ be the image of $\overline{\mathfrak{u}}_{\alpha_{i}}$ in $\mathfrak{s p i n}_{2 n+1}\left(A\left[\frac{1}{p}\right]\right)$. Let $\overline{\mathfrak{u}}_{\alpha_{\bullet}}^{A, j}$ the sequence defined by $\overline{\mathfrak{u}}_{\alpha_{\bullet}}^{A, 1}=\overline{\mathfrak{u}}_{\alpha_{1}}^{A}, \overline{\mathfrak{u}}_{\alpha_{\bullet}}^{A, 2}=\left[\overline{\mathfrak{u}}_{\alpha_{1}}^{A}, \overline{\mathfrak{u}}_{\alpha_{2}}^{A}\right]$, and for $j \leq n-1$ :

$$
\overline{\mathfrak{u}}_{\alpha \bullet}^{A, j+1}=\left[\overline{\mathfrak{u}}_{\alpha_{\bullet}}^{A, j}, \overline{\mathfrak{u}}_{\alpha_{j+1}}^{A}\right]
$$

Note that by calculations in the proof of Theorem $4.8, \overline{\mathfrak{u}}_{\alpha \bullet}^{A, n}$ is an $A\left[\frac{1}{p}\right]$-module.

Lemma 4.10. For any $n \geq 1$, assume $\rho_{\theta}$ is full over $\Lambda_{n}$. For any characteristic zero quotient $A$ of $\Lambda_{n}$, there exists a unique Lie subalgebra $\operatorname{Lie}_{A}$ of the $\mathbb{Q}_{p}$-Lie algebra $\operatorname{Lie}_{\mathbb{Q}_{p}} \operatorname{Im} \rho_{A}$ which is an $A \otimes \mathbb{Q}_{p}$-Lie algebra, such that $\operatorname{Lie}_{A} \cap \overline{\mathfrak{u}}_{\sum_{i} \alpha_{i}}^{A}=\overline{\mathfrak{u}}_{\alpha_{\bullet}}^{A, n}$ and which is stable by the adjoint action of Im $\rho_{A}$. If $\Lambda_{n} \rightarrow A \rightarrow B$ are two characteristic zero quotients of $\Lambda_{n}$, then $\mathrm{Lie}_{A} \rightarrow \mathrm{Lie}_{B}$ is surjective.

Note however that for some quotients $A$, this algebra may be zero because $\overline{\mathfrak{u}}_{\alpha \bullet}^{A, n}$ may be zero, although it is not the case over $\Lambda_{n}$. This is why we'll need a modified construction later (see Lemma $5.4)$.

Proof. To simplify notations, we prove it for $\mathfrak{s p}_{4}$. Let $\alpha_{1}=t_{1} t_{2}^{-1}$ and $\alpha_{2}=t_{2}^{2}$ be the two simple roots. We form $\overline{\mathfrak{u}}_{\alpha_{\bullet}}^{A, 2}=\left[\overline{\mathfrak{u}}_{\alpha_{1}}^{A}, \overline{\mathfrak{u}}_{\alpha_{2}}^{A}\right]$ which is an $A$-module by calculations at the end of the proof of Theorem 4.8. If it is non zero, by Poisson bracket of $\overline{\mathfrak{u}}_{\alpha}^{A, 2}$ with all the $\overline{\mathfrak{u}}_{\gamma}^{A}$, s for all the root (positive or negative) of $\mathfrak{s p}_{4}$, we construct $A\left[\frac{1}{p}\right]$-submodules $\overline{\mathfrak{u}}_{\alpha_{1}+\alpha_{2}+\gamma}^{A,}$ of $\overline{\mathfrak{u}}_{\alpha_{1}+\alpha_{2}+\gamma}^{A}$. Note that in particular, $\overline{\mathfrak{u}}_{\alpha_{1}+\alpha_{2}}^{A, \prime}=\overline{\mathfrak{u}}_{\alpha_{1}+\alpha_{2}}^{A}$. We also define a Cartan subalgebra $\overline{\mathfrak{t}}^{A, \prime}$ by $\left[\overline{\mathfrak{u}}_{\alpha_{1}}^{A, \prime}, \overline{\mathfrak{u}}_{-\alpha_{1}}^{A, \prime}\right] \oplus\left[\overline{\mathfrak{u}}_{\alpha_{2}}^{A, \prime}, \overline{\mathfrak{u}}_{-\alpha_{2}}^{A, \prime}\right]$, and we then define an $A \otimes \mathbb{Q}_{p}$-module by

$$
\operatorname{Lie}_{A}=\overline{\mathfrak{t}}^{A, \prime} \oplus \bigoplus_{\gamma} \overline{\mathfrak{u}}_{\gamma}^{A, \prime}
$$

Using the Jacobi identity for the Poisson bracket, one sees easily that it is an $A \otimes \mathbb{Q}_{p}$-Lie algebra which has all the desired properties.

It follows from the construction above that if $\rho_{\theta}$ is full over $\Lambda_{n}$; then for all roots $\gamma, \overline{\mathfrak{u}}_{\gamma}^{\prime}$ which is the $\gamma(\delta)$-eigenspace for the action of $\delta$ as in Theorem 4.8) on $\operatorname{Lie}_{\Lambda_{n}}$, is non zero. More precisely,

Corollary 4.11. If $\rho_{\theta}$ is full over $\Lambda_{n}$, there exists a non zero largest $\Lambda_{n}\left[\frac{1}{p}\right]$-ideal $\mathfrak{l}_{\theta}^{\prime}$ such that

$$
\mathfrak{l}_{\theta}^{\prime} \cdot \mathfrak{s p}_{4}\left(\Lambda_{n} \otimes \mathbb{Q}_{p}\right) \subset \operatorname{Lie}_{\Lambda_{n}}
$$

We have $\mathfrak{l}_{\theta}^{\prime} \subset \mathfrak{l}_{\theta} \otimes \mathbb{Q}_{p}$ and these two ideals have the same radical : $\sqrt{\mathfrak{r}_{\theta}^{\prime}}=\sqrt{\mathfrak{r}_{\theta} \otimes \mathbb{Q}_{p}}$ in $\Lambda_{n}\left[\frac{1}{p}\right]$.

\section{Galois level and Congruence Ideals}

5.1. Congruence ideals. Let $\theta: \mathbf{T}^{M \text {,holcusp }} \rightarrow \mathbb{I}_{0}$ be an irreducible component of Spec $\mathbf{T}^{M \text {,holcusp }}$; we denote by $\mathbb{I}$ the normalization of $\mathbb{I}_{0}$ and by $\mathcal{K}$ its field of fractions; let $\mathbf{T}_{\mathbb{I}}=\mathbf{T}^{M \text {,holcusp }} \otimes_{\Lambda_{n}} \mathbb{I}$ and $\theta_{\mathbb{I}}=m_{\mathbb{I}} \circ \theta \otimes \operatorname{Id}_{\mathbb{I}}$ the composition of the extension of scalars with the multiplication on $\mathbb{I}$; by the diagonalisability of $\mathbf{T}^{M \text {,holcusp }}$ over $\mathcal{K}$, the character $\theta_{\mathbb{I}}: \mathbf{T}_{\mathbb{I}} \rightarrow \mathbb{I}$ splits over $\mathcal{K}$, and there is an isomorphism of $\mathcal{K}$-algebras :

$$
\mathbf{T}_{\mathbb{I}} \otimes_{\mathbb{I}} \mathcal{K} \cong \mathcal{K} \times \mathbf{T}_{\mathcal{K}}^{\prime}
$$

with the first projection $\operatorname{pr}_{1}$ given by $\theta_{\mathcal{K}}=\theta_{\mathbb{I}} \otimes \mathcal{K}$.

(Mult 1) We assume that $\theta_{\mathbb{I}}$ does not factor through $\mathrm{pr}_{2}$. In other words, the family $\theta_{\mathbb{I}}$ occurs with multiplicity 1 in $\mathbf{T}_{\mathbb{I}}$.

If $n=1$, this assumption amounts to saying that the family $\theta_{\mathbb{I}}$ is $M$-new (i.e. all the forms occurring in the family are $M$-new); if $n=2$, using the Roberts-Schmidt theory of newforms, and assuming a folklore conjecture which says that automorphic forms on $\mathrm{GSp}_{4}$ which are neither CAP nor endoscopic satisfy multiplicity one, one can prove that it holds for $M$-new families of squarefree level $M$. For $n \geq 3$, we simply assume (Mult 1).

Let $\mathbf{T}^{\prime}$ be the image of $\mathbf{T}_{\mathbb{I}}$ by the second projection $\mathrm{pr}_{2}$. We define then $\mathfrak{c}_{1}=\mathbf{T}_{\mathbb{I}} \cap(\mathbb{I} \times\{0\})$, which is an ideal of $\mathbf{T}_{\mathbb{I}}$ and of $\mathbb{I} \times\{0\}$, and $\mathfrak{c}_{2}=\mathbf{T}_{\mathbb{I}} \cap\left(\{0\} \times \mathbf{T}^{\prime}\right)$ which is an ideal of $\mathbf{T}_{\mathbb{I}}$ and of $\times \mathbf{T}^{\prime} \times\{0\}$. Note that $\mathfrak{c}_{1}=\operatorname{Ker} \operatorname{pr}_{2}$ and $\mathfrak{c}_{2}=\operatorname{Ker} \operatorname{pr}_{1}$, and that for $i=1,2$, one can identify $\mathfrak{c}_{i}$ with its image $\operatorname{pr}_{i}\left(\mathfrak{c}_{i}\right)$. We also define $\mathfrak{c}=\mathfrak{c}_{1}+\mathfrak{c}_{2}$. The projections $\operatorname{pr}_{i}$ induce isomorphisms of $\mathbb{I}$-modules

$$
\overline{\mathrm{pr}}_{1}: \mathbf{T}_{\mathbb{I}} / \mathfrak{c} \rightarrow \mathbb{I} / \mathfrak{c}_{1} \text { and } \quad \overline{\mathrm{pr}}_{2}: \mathbf{T}_{\mathbb{I}} / \mathfrak{c} \rightarrow \mathbf{T}^{\prime} / \mathfrak{c}_{2}
$$


We thus obtain the congruence isomorphism $\overline{\operatorname{pr}}_{2} \circ \overline{\operatorname{pr}}_{1}^{-1}: \mathbb{I} / \mathfrak{c}_{1} \cong \mathbf{T}^{\prime} / \mathfrak{c}_{2}$.

Definition 5.1. The ideal $\mathfrak{c}_{\theta}=\mathfrak{c}_{1}$ is called the congruence ideal associated to the family $\theta$.

For any prime $P$ of $\mathbb{I}$ containing $\mathfrak{c}_{\theta}$, there exists another family $\theta^{\prime}: \mathbf{T}_{\mathbb{I}} \rightarrow \mathbb{J}$ (for some finite normal extension $\mathbb{J}$ of $\mathbb{I})$ and a prime $Q$ of $\mathbb{J}$ above $P$ such that $\theta_{\mathbb{I}} \cong \theta^{\prime}(\bmod Q)$.

Let $\theta_{2}: \mathbf{T}^{M \text {,holcusp }} \rightarrow \mathbb{J}$ be a second family, different from $\theta$, hence it factors through $\mathrm{pr}_{2}$. Let $\mathfrak{c}_{\theta_{2}} \subset \mathbb{J}$ be its congruence ideal ; if $Q$ is a prime of $\mathbb{J}$ containing both the ideals $\mathfrak{c}_{\theta}$ and $\mathfrak{c}_{\theta_{2}}$, and if $P=\mathbb{I} \cap Q$, the congruence isomorphism induces a congruence injection $\mathbb{I} / P \cong \mathbb{J} / Q$ which expresses a congruence between the two specific families $\theta$ and $\theta_{2}$ modulo $Q$. Therefore the intersection locus

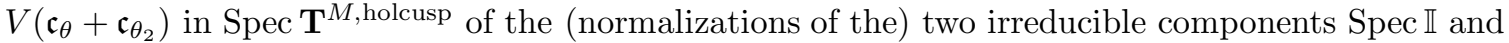
Spec $\mathbb{J}$ is also the congruence locus of the two families $\theta$ and $\theta_{2}$. Note that this locus is empty unless both families factor through the same local factor of the semilocal algebra $\mathbf{T}^{M \text {,holcusp }}$; in other words a necessary condition for this locus to be non empty is that the residual representations $\bar{\rho}_{\theta}$ and $\bar{\rho}_{\theta_{2}}$ coincide.

5.2. Comparison between Galois level and congruence ideals. In this section we assume again that the family $\theta: \mathbf{T}^{M, \text { holcusp }} \rightarrow \mathbb{I}$ is $\Lambda_{n}$-valued, i.e. $\mathbb{I}=\Lambda_{n}$. We also assume that its residual Galois representation $\bar{\rho}_{\theta}$ is irreducible and that $\rho=\rho_{\theta}$ satisfies the assumptions of Theorem 4.8 : there is an arithmetic specialization for which $\operatorname{Im} \rho_{P_{k 0}}$ is full and that there is a $\mathbb{Z}_{p}$-regular element $\delta$ in $\operatorname{Im} \rho$. By Theorem 4.8, the representation $\rho_{\theta}$ is full; we denote by $\mathfrak{l}_{\theta}$ the Galois level of $\theta$, that is, the largest ideal of $\Lambda_{n}$ such that $\Gamma_{\Lambda_{n}}\left(\mathfrak{l}_{\theta}\right) \subset \operatorname{Im} \rho_{\theta}$.

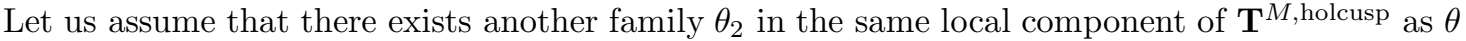
(so that its residual representation coincides with that of $\theta$, in particular it is irreducible) but such that $\rho_{\theta_{2}}$ is not full. Let $\mathfrak{c}\left(\theta, \theta_{2}\right)=\mathfrak{c}_{\theta} \cdot \mathbb{J}+\mathfrak{c}_{\theta_{2}}$ be the ideal of $\mathbb{J}$ defining the congruence locus between $\theta$ and $\theta_{2}$ and let $c(\theta)=\bigcap_{\theta_{2}} \mathfrak{c}\left(\theta, \theta_{2}\right) \cap \Lambda_{n}$, where the intersection of the $\mathfrak{c}\left(\theta, \theta_{2}\right)$ 's is taken over all the families $\theta_{2}$ congruent to $\theta$ but not full. Let $Q$ be a prime ideal of $\mathbb{J}$ containing $\mathfrak{c}\left(\theta, \theta_{2}\right)$, and let $P=Q \cap \Lambda_{n}$. Then, we have a congruence $\rho_{\theta} \equiv \rho_{\theta_{2}}(\bmod Q)$; then $\rho_{\theta}(\bmod P)$ is not full ; hence we must have $\mathfrak{l}_{\theta} \subset Q$. Since for any prime $P$ of $\Lambda_{n}$ containing $c(\theta)$ there exists a prime $Q$ above $P$ in a finite extension which contains $\bigcap_{\theta_{2}} \mathfrak{c}\left(\theta, \theta_{2}\right)$, we see that any prime ideal $P$ containing $c(\theta)$ contains $\mathfrak{l}_{\theta}$. For any ideal $\mathfrak{a}$ of $\Lambda_{n}$, let $V(\mathfrak{a})$ be the set of prime ideals containing $\mathfrak{a}$. Recall that in the case $n=1$, Hida has proved in $[\mathrm{H} 13 \mathrm{~b}]$ that if the family $\theta$ is not CM but there is another family $\theta_{2}$ which is CM for a given imaginary quadratic field, then the sets $V\left(\mathfrak{l}_{\theta}\right)$ and $V(c(\theta))$ coincide; actually under slightly stronger assumptions (see [H13b, Th.7.2]), he could use Pink's Lie algebra theory for $\mathrm{SL}_{2}$ to compare the exponents of the height one primes occurring in (the reflexive envelopes of) the two ideals $\mathfrak{l}_{\theta}$ and $c(\theta)$, see [H13b, Th.8.5.].

For general $n$, a natural question is to ask whether the converse is true, namely that if a prime $P$ divides $\mathfrak{l}_{\theta}$, there exists a non full family $\theta_{2}$ congruent to $\theta$ at a prime $Q$ above $P$. Assume again $n=2$, recall that given a real quadratic field $F$ of discriminant $D$, and a Hilbert modular cusp form for $F$ of level $\mathfrak{n}$ and weight $\left(m_{1}, m_{\sigma}\right)$ with $m_{1}=m_{\sigma}+2 r$ with $r \geq 1$ and $m_{\sigma} \geq 2$, eigen for the Hecke operators and p-ordinary, one can define its theta lift to GSp 4 (see [Y79] and [R01, Th.8.6]), which

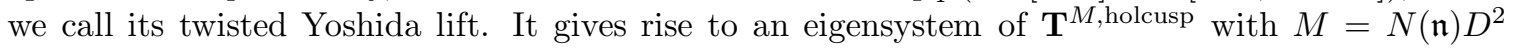
of weight $\left(k_{1}, k_{2}\right)$ with $k_{1}=m_{\sigma}+r$ and $k_{2}=r+2$, (so, $k_{1} \geq k_{2} \geq 3$ ), see for instance [MT02, Section 7.3]. Assuming moreover that $\mathfrak{n}$ is prime to $p$ and that $g$ is $p$-ordinary, there exists a unique 2 -variable $p$-adic family of $p$-ordinary Hilbert modular forms $\mathbf{g}$ of auxiliary level $\mathfrak{n}$ passing through $g$ (after $p$-stabilization). It defines a homomorphism of $\Lambda_{2}$-algebras $\theta_{\mathbf{g}}: \mathbf{T}_{F}^{\mathfrak{n}} \rightarrow \mathbb{J}$ of the ordinary Hida Hecke algebra $\mathbf{T}_{F}^{\mathfrak{n}}$ for Hilbert cusp forms over $F$, of auxiliary level $\mathfrak{n}$. For any place $w$ of $F$ prime to $\mathfrak{n} p$, this homomorphism sends the Hecke operator $T_{w}$ to the eigenvalue $\theta_{\mathbf{g}}\left(T_{w}\right)$ on $\mathbf{g}$. The map $\left(m_{1}, m_{\sigma}\right) \mapsto\left(k_{1}, k_{2}\right)$ defines an automorphism of $\Lambda_{2}$, denoted by $\tau$.

Lemma 5.2. Given $(F, \mathbf{g})$ of a real quadratic field and a 2-variable family of p-ordinary cusp forms $\theta_{\mathbf{g}}: \mathbf{T}_{F}^{\mathfrak{n}} \rightarrow \mathbb{J}$, then, for $M=N(\mathfrak{n}) D^{2}$, there exists a 2-variable p-adic family $\theta_{2}: \mathbf{T}^{M, \text { holcusp }} \rightarrow \mathbb{J}^{\tau}$, which is a homomorphism of $\Lambda_{2}$-algebras via $\tau: \theta_{2}(\alpha \cdot T)=\tau(\alpha) \cdot \theta_{2}(T)$, such that for every prime $q$ not dividing $M p$,

$$
\theta_{2}\left(T_{q, 1}\right)=\lambda_{q}(\mathbf{g}) \text { and } \quad \theta_{2}\left(T_{q, 2}\right)=\mu_{q}(\mathbf{g})
$$

where the Hecke eigenvalues $\lambda_{q}(\mathbf{g})$ and $\mu_{q}(\mathbf{g})$ are defined as follows 
- If $q$ splits in $F$, let $w_{1}$ and $w_{2}$ the places above $q$, then $\lambda_{q}(\mathbf{g})=\theta_{\mathbf{g}}\left(T_{w_{1}}\right)+\theta_{\mathbf{g}}\left(T_{w_{2}}\right)$ and $q \cdot \mu_{q}(\mathbf{g})=q^{2}+q \theta_{\mathbf{g}}\left(T_{w_{1}}\right) \theta_{\mathbf{g}}\left(T_{w_{2}}\right)-1$

- If $q$ is inert, $\lambda_{q}(\mathbf{g})=0$ and $q \cdot \mu_{q}(\mathbf{g})=-\left(q^{2}+q \theta_{\mathbf{g}}\left(T_{w}\right)+1\right)$

We call $\theta_{2}=\theta_{2}(F, \mathbf{g})$ the twisted Yoshida lift of $(F, \mathbf{g})$

The proof is by comparing for each prime $q$ not dividing $M p$ the characteristic polynomial of $\operatorname{Ind}_{G_{F}}^{G_{Q}} \rho_{h}$ for all classical Hilbert forms $h$ in the family $\mathbf{g}$ and the GSp 4 -Hecke polynomial at $q$ of its Yoshida lift.

From now on, we fix a real quadratic field $F$ of discriminant $D$, an auxiliary level $\mathfrak{n}$ in $F$ prime to $p$ and we pose $M=N(\mathfrak{n}) D^{2}$; we give ourselves a $\Lambda_{2}$-valued family $\theta: \mathbf{T}^{M \text {, holcusp }} \rightarrow \Lambda_{2}$ which satisfies the assumptions of $\S 4.3$, so that its Galois representation $\rho_{\theta}: G_{\mathbb{Q}} \rightarrow \operatorname{GSp}_{4}\left(\Lambda_{2}\right)$ is full.

In the following theorem, we assume there exists a Hilbert modular form $g$ of weight $m_{1}=m_{\sigma}+2 r$ with $r \geq 1$ (hence the form $g$ does not descend to $\mathbb{Q}$ ) and $m_{\sigma} \geq 2$, such that

- $\bar{\rho}_{g}$ is "full" : that is, $\mathrm{SL}_{2}\left(\mathbb{F}_{p}\right) \subset \operatorname{Im} \bar{\rho}_{g}$,

- there exists no character $\xi: G_{\mathbb{Q}} \rightarrow \overline{\mathbb{F}}_{p}^{\times}$such that $\bar{\rho}_{g^{\sigma}} \cong \bar{\rho}_{g} \otimes \xi$

We finally assume that $\bar{\rho}_{\theta}=\operatorname{Ind}_{G_{F}}^{G_{Q}} \bar{\rho}_{g}$. By the previous lemma, there exists a "twisted Yoshida lift" family $\theta_{2}=\theta_{2}(F, \mathbf{g})$ in the same local component of $\mathbf{T}^{M \text {,holcusp }}$ as $\theta$. Note that if there is another quadratic field $F^{\prime}$ and a Hilbert form $g^{\prime}$ on $F^{\prime}$ such that $\bar{\rho}_{\theta}=\operatorname{Ind}_{G_{F^{\prime}}}^{G_{Q}} \bar{\rho}_{g^{\prime}}$ then $F^{\prime}=F$. Indeed, if this equality holds and $F^{\prime} \neq F$, we have an isomorphism of $G_{\mathbb{Q}}$-modules $\bar{\rho}_{\theta} \otimes \xi \cong \bar{\rho}_{\theta}$ where $\xi$ is the (non trivial) quadratic character associated to $F^{\prime}$; hence, by irreducibility of the $G_{F}$-modules $\bar{\rho}_{g}$ and $\bar{\rho}_{g^{\sigma}}$, we have an isomorphism of $G_{F^{-}}$modules $\bar{\rho}_{g} \otimes \xi \cong \bar{\rho}_{g}$ (which implies that $\bar{\rho}_{g} \cong \operatorname{Ind}_{F_{F^{\prime}}}^{F} \varphi$ for a character $\varphi$ and is impossible by fullness of $\bar{\rho}_{g}$ ) or $\bar{\rho}_{g} \otimes \xi \cong \bar{\rho}_{g^{\sigma}}$ which is impossible by assumption.

Theorem 5.3. For $n=2$, assume that $\rho_{\theta}$ satisfies the assumptions of Theorem 4.8 , hence is full, and that there is a Hilbert cusp form $g$ for $F$ as above such that $\bar{\rho}_{\theta}=\operatorname{Ind}_{G_{F}}^{G_{Q}} \bar{\rho}_{g}$, then we have $V\left(c(\theta)\left[\frac{1}{p}\right]\right)=V\left(\mathfrak{l}_{\theta}\left[\frac{1}{p}\right]\right)$.

Note that we are not yet able to study the prime $(p)$; more precisely, we can't prove that if $(p) \in V\left(\mathfrak{l}_{\theta}\right)$, then $(p) \in V(c(\theta))$. We need first a variant of 4.10 and 4.11 , because in our case 4.10 applied to the quotient $A$ of interest for us yields a trivial $A \otimes \mathbb{Q}_{p}$-Lie algebra. Since $\rho_{\theta}$ is congruent to $\operatorname{Ind}_{G_{F}}^{G_{Q}} \rho_{g}$ and since its image contains the diagonal element $\delta$ with distinct eigenvalues, we see that the diedral group generated by $\delta$ and by the element $w$ of the Weyl group which exchanges $t_{1}$ and $t_{2}$ in $\operatorname{diag}\left(t_{1}, t_{2}, t_{2}^{-1}, t_{1}^{-1}\right)$ lifts to characteristic zero as a subgroup of $\operatorname{Im} \rho_{\theta}$ (by a theorem of $\mathrm{P}$. Hall, as the kernel of $\operatorname{Im} \rho_{\theta} \rightarrow \operatorname{Im}_{\operatorname{Ind}_{G_{F}}^{G_{Q}} \rho_{g}}$ is pro- $p$ ); hence $\operatorname{Im} \rho_{\theta}$ contains the element $w$ of the Weyl group which exchanges $t_{1}$ and $t_{2}$. Let $\alpha_{1}=t_{1} t_{2}^{-1}$ and $\alpha_{2}=t_{2}^{2}$ be the two simple roots. We recall the notations $\overline{\mathfrak{u}}_{\gamma}^{A}=\mathfrak{u}_{\gamma}(A) \cap \operatorname{Lie}_{\mathbb{Q}_{p}} \operatorname{Im} \rho_{A}$ for all roots $\gamma$ 's.

Lemma 5.4. For any characteristic zero quotient $A$ of $\Lambda_{2}$, there exists a Lie subalgebra $\operatorname{Lie}_{A}$ of the $\mathbb{Q}_{p}$-Lie algebra $\operatorname{Lie}_{\mathbb{Q}_{p}} \operatorname{Im} \rho_{A}$ which is an $A \otimes \mathbb{Q}_{p}$-Lie algebra, which contains $\overline{\mathfrak{u}}_{\alpha_{2}}^{A}$ and which is stable by the adjoint action of $\operatorname{Im} \rho_{A}$. If $\Lambda_{2} \rightarrow A \rightarrow B$ are two characteristic zero quotients of $\Lambda_{2}$, then $\mathrm{Lie}_{A} \rightarrow \mathrm{Lie}_{B}$ is surjective.

Proof. By action of the inertia as in the proof of Theorem 4.8, we see that $\overline{\mathfrak{u}}_{\alpha_{2}}$, resp. $\overline{\mathfrak{u}}_{2 \alpha_{1}+\alpha_{2}}$, carries an action of $\left(1+T_{1}\right)\left(1+T_{2}\right)^{-1}$, resp. of $\left(1+T_{1}\right)\left(1+T_{2}\right)$; moreover $\overline{\mathfrak{u}}_{\alpha_{2}}=w\left(\overline{\mathfrak{u}}_{2 \alpha_{1}+\alpha_{2}}\right)$ hence $\overline{\mathfrak{u}}_{\alpha_{2}}$ carries a structure of $A$-module by transport of structure. We construct $\mathrm{Lie}_{A}$ as the Lie algebra generated by $\overline{\mathfrak{u}}_{\alpha_{2}}^{A}, \overline{\mathfrak{u}}_{2 \alpha_{1}+\alpha_{2}}$ and their Poisson brackets by the $\overline{\mathfrak{u}}_{\alpha_{2}}^{A}$ 's for all the root (positive or negative) of $\mathfrak{s p}_{4}$. This defines the desired $A \otimes \mathbb{Q}_{p}$-Lie algebra.

Again, it follows from the construction above that if $\rho_{\theta}$ is full over $\Lambda_{2}$; then for all roots $\gamma$, the $\gamma(\delta)$-eigenspace for the action of $\delta$ as in Theorem 4.8) on $\mathrm{Lie}_{\Lambda_{2}}$, is non zero. More precisely,

Corollary 5.5. If $\rho_{\theta}$ is full over $\Lambda_{2}$, there exists a non zero largest $\Lambda_{n}\left[\frac{1}{p}\right]$-ideal $\mathfrak{l}_{\theta}^{\prime}$ such that

$$
\mathfrak{l}_{\theta}^{\prime} \cdot \mathfrak{s p}_{4}\left(\Lambda_{n}\left[\frac{1}{p}\right]\right) \subset \operatorname{Lie}_{\Lambda_{2}}
$$

We have $\mathfrak{l}_{\theta}^{\prime} \subset \mathfrak{l}_{\theta} \otimes \mathbb{Q}_{p}$ and these two ideals have the same radical $: \sqrt{\mathfrak{r}_{\theta}^{\prime}}=\sqrt{\mathfrak{l}_{\theta} \otimes \mathbb{Q}_{p}}$. 
Proof. Simply review the construction of $\operatorname{Lie}_{\Lambda_{2}}$ starting from $\mathfrak{u}_{\gamma}\left(\mathfrak{l}_{\theta}\right) \subset \overline{\mathfrak{u}}_{\gamma}$ for all $\gamma$ 's, and take Poisson brackets.

Proof of the theorem. Let us prove that if $P$ is a characteristic zero prime containing $\mathfrak{l}_{\theta}$, then it contains $c(\theta)$. We can assume that $P$ is an isolated component in the primary decomposition of the ideal $\mathfrak{l}_{\theta}^{\prime}$ defined in the previous corollary. Let $\mathfrak{q}$ be the primary component of $\mathfrak{l}_{\theta}^{\prime}$ whose radical is $P$. Let $\Lambda_{P}$ be the localization of $\Lambda_{2}$ at $P, \kappa_{P}=\operatorname{Frac}\left(\Lambda_{2} / P\right)$ its residue field. Let $\widetilde{\Lambda}=\Lambda_{2} / \mathfrak{q}$ and $\widetilde{\Lambda}_{P}$ its localization at $P$; it is an artinian $\Lambda_{P}$-algebra with residue field $\kappa_{P}$.

Let $\widetilde{\rho}$ be the reduction of $\rho_{\theta}$ modulo $\mathfrak{q}$; it takes values in $G(\widetilde{\Lambda})$. We consider an ideal $\mathfrak{a}$ of $\Lambda_{2}$ containing $\mathfrak{q}$ and $\tilde{\mathfrak{a}}=\mathfrak{a} / \mathfrak{q}$, such that $\tilde{\mathfrak{a}}_{P}$ is one-dimensional over $\kappa_{p}$. Consider the $\widetilde{\Lambda} \otimes \mathbb{Q}_{p}$-Lie algebra $\operatorname{Lie}_{\widetilde{\Lambda}}$ of Lemma 5.4 in $\mathfrak{g s p}_{4}(\widetilde{\Lambda})$ and the Lie subalgebra $\mathfrak{s}=\widetilde{\mathfrak{a}} \cdot \mathfrak{s p}_{4}(\widetilde{\Lambda}) \cap \operatorname{Lie}_{\widetilde{\Lambda}}$ inside the Lie algebra $\widetilde{\mathfrak{a}} \cdot \mathfrak{s p}_{4}(\widetilde{\Lambda})$, after localization at $P$, the latter is isomorphic to $\mathfrak{s p}_{4}\left(\kappa_{P}\right)$. So we can identify $\mathfrak{s}_{P}$ to a $\kappa_{P}$-Lie subalgebra of $\mathfrak{s p}_{4}\left(\kappa_{P}\right)$. Let us prove that $\mathfrak{s}_{P} \neq \mathfrak{s p}_{4}\left(\kappa_{P}\right)$. Indeed if equality holds, we have $\widetilde{\mathfrak{a}} \cdot \mathfrak{s p}_{4}(\widetilde{\Lambda}) \subset \operatorname{Lie}_{\widetilde{\Lambda}}$. hence $\mathfrak{a} \cdot \mathfrak{s p}_{4}\left(\Lambda_{2}\right) \subset \operatorname{Lie}_{\Lambda_{2}}+\mathfrak{q} \cdot \mathfrak{s p}_{4}\left(\Lambda_{2}\right)$ so after localizing at $P$, noting that $\mathfrak{l}_{\theta, P}^{\prime}=\mathfrak{q}_{P}$ and that $\mathfrak{l}_{\theta}^{\prime} \cdot \mathfrak{s p}_{4}\left(\Lambda_{2}\right) \subset \operatorname{Lie}_{\Lambda_{2}}$, we have $\mathfrak{a}_{P} \cdot \mathfrak{s p}_{4}\left(\Lambda_{P}\right) \subset \operatorname{Lie}_{\Lambda_{P}}$. Consider the ideal $\mathfrak{b}=\mathfrak{l}_{\theta}^{\prime}+\mathfrak{a}$, it is strictly larger than $\mathfrak{l}_{\theta}^{\prime}$ and we have $\mathfrak{b} \cdot \mathfrak{s p}_{4}\left(\Lambda_{2}\right) \subset \operatorname{Lie}_{\Lambda_{2}}$; this contradicts the definition of $\mathfrak{l}_{\theta}^{\prime}$.

In conclusion, $\mathfrak{s}_{P}$ is a strict $\kappa_{P}$-Lie subalgebra of $\mathfrak{s p}_{4}\left(\kappa_{P}\right)$. It is semisimple because $\widetilde{\rho}$ is irreducible. Hence it is either 0 , or an $\mathfrak{s l}_{2}$ in the Levi of a maximal parabolic, or $\mathrm{Sym}^{3} \mathfrak{s l}_{2}$, or the endoscopic $\mathfrak{s l}_{2} \times \mathfrak{s l}_{2}$. Since it is normalized by $\operatorname{Im} \rho_{P}$ (recall $\rho_{P}$ is the representation over $\Lambda_{2} / P$ ), $\operatorname{Im} \rho_{P}$ is contained in the corresponding normalizer. However the second and third cases are impossible. Indeed, for any root $\gamma$ occurring in $\mathfrak{s l}_{2} \times \mathfrak{s l}_{2}$, one can show using the projection $\Delta_{\gamma}$ as Lemma 4.5 and Lemma 4.7, that $U_{\gamma}(\widetilde{\Lambda}) \cap \operatorname{Im} \widetilde{\rho}$ maps surjectively to $U_{\gamma}\left(\mathbb{F}_{p}\right) \cap \operatorname{Im} \bar{\rho}$. Hence by the assumption of fullness of $\bar{\rho}_{g}$ and $\bar{\rho}_{g^{\sigma}}$, these groups are non trivial. For instance for $\gamma=\alpha_{2}$, by taking a power of $p$ and taking the Log of a non trivial element, we find a non trivial element of $\overline{\mathfrak{u}}_{\alpha_{2}}^{\widetilde{\Lambda}} \subset$ Lie $_{\tilde{\Lambda}}$, since both sides are $\widetilde{\Lambda}$-modules, one can assume that this non zero element is in $\overline{\mathfrak{u}}_{\alpha_{2}}^{\widetilde{\Lambda}}(\widetilde{\mathfrak{a}}) \subset$ Lie $_{\widetilde{\Lambda}}$, hence $\mathfrak{s}_{P} \neq 0$. The only remaining case is the endoscopic $\mathfrak{s l}_{2} \times \mathfrak{s l}_{2}$ and $\left.\operatorname{Im} \rho_{P} \subset N\left(\mathrm{GL}_{2} \times \mathrm{GL}_{2}\right)^{\circ}\right)$. Comparing with the reduction modulo the maximal ideal, we find a two-dimensional Galois representation of $G_{F}$ over $\Lambda_{2} / P$ congruent to $\bar{g}$ modulo the maximal ideal of $\Lambda_{2} / P$.

From this, one can deduce, using an $R_{F}=\mathbf{T}_{F}$ of Skinner-Wiles [SW01], that there exists a Hilbert family $\mathbf{g}$ such that the family $\theta_{2}: \mathbf{T}^{M, \text { holcusp }} \rightarrow \mathbb{J}$ associated to $(F, \mathbf{g})$ which is congruent to $\theta$ modulo a prime $Q$ of $\mathbb{J}$ above $P$.

Questions : 1) By this theorem, we can ask more precisely about the primary ideals (for minimal primes) occurring in $c(\theta)\left[\frac{1}{p}\right]$ and $\mathfrak{l}_{\theta}\left[\frac{1}{p}\right]$. Are they equal?

2) Note that for a Hilbert form $g$ on a real quadratic field $F$, the $L$ function of $\operatorname{Ad}_{\operatorname{Ind}_{G_{F}}}^{G_{Q}} \rho_{g}$ can be decomposed as

$$
L\left(\operatorname{Ad} \operatorname{Ind}_{G_{F}}^{G_{\mathbb{Q}}} \rho_{g}, s\right)=L\left(\operatorname{Ind}_{G_{F}}^{G_{\mathbb{Q}}} \operatorname{Ad} \rho_{g}, s\right) L\left(R_{g}, s\right)
$$

where $R_{g}$ is the extension to $G_{\mathbb{Q}}$ of the representation $\rho_{g} \otimes \rho_{g^{\sigma}}^{\vee}$ of $G_{F}$ appearing as a factor of $\operatorname{Ad} \operatorname{Ind}_{G_{F}}^{G_{\mathbb{Q}}} \rho_{g}$.

By a theorem of [Di05], the normalized special value at 1 of the first factor control congruences between the Hilbert form $g$ and other Hilbert forms, hence a similar normalized special value at 1 of the second factor should control the congruences between the Yoshida lift of $g$ and other Siegel forms which are not theta lifts of Hilbert forms. The $p$-adic $L$ function interpolating the values of the first factor for $g$ varying in a Hida family $\mathrm{g}$ has been defined by Hida. It might be possible to define the $p$-adic $L$ function interpolating the second factor for $g$ varying in a Hida family. Then one could ask how it is related to the determination of $\mathfrak{l}_{\theta}$ for a family $\theta$ congruent to $\Theta(\mathbf{g})$.

3) More generally, we hope to treat other cases of congruences between a "full" family and other families of automorphic forms with "small but irreducible Galois image", for GSp(4) and other groups.

\section{REFERENCES}

[Ca13] F. Calegari, Even Galois representations and the Fontaine-Mazur conjecture, Invent math. (DOI $10.1007 / \mathrm{s} 00222-010-0297-0)$ 
[CaGe13] F. Calegari, T. Gee, Irreducibility of automorphic Galois representations of GL( $n), n$ at most 5 , to appear in Ann. Inst. Fourier

[Car94] H. Carayol, Formes modulaires et représentations galoisiennes à valeurs dans un anneau local complet, in "p-adic monodromy and the Birch and Swinnerton-Dyer conjecture", Contemp. Math. vol.165, AMS 1994, pp. 213-237

[Che51] C. Chevalley, Théorie des groupes de LIE, II et III, Publ. Inst. Math. Univ. Nancago, Paris, Hermann 1951 et 1955

[Ch92] W.-C. Chi, $l$-adic and $\lambda$-adic representations associated to abelian varieties defined over number fields. Amer. J. Math. 114 (1992), 315-353.

[Di05] M. Dimitrov, On Ihara's Lemma for Hilbert modular varieties, Compositio Mathematica 145, Issue 05 (2009), 1114-1146.

[Em14] M. Emerton, Local-global compatibility in the $p$-adic Langlands programme for $\mathrm{GL}_{2 / \mathbb{Q}}$, preprint

[FC90] G. Faltings, C.-L. Chai, Degeneration of Abelian Varieties, Erg. Math. Series 3-22, Springer Verlag 1990

[H02] H. Hida, Control theorems of coherent sheaves on Shimura varieties of PEL type, J. Inst. Math. Jussieu, 1, 2002, pp.1-76

[H13a] H. Hida, Image of $\Lambda$-adic Galois representations modulo $p$, Invent. Math. 194 (2013), 1-40.

[H13b] H. Hida, Big Galois representations and $p$-adic $L$-functions, preprint, 2012, 51 pages, to appear in Compositio Math.

[Lau05] G. Laumon, Fonctions zêta des variétés de Siegel de dimension trois, in Formes Automorphes (II), le cas du groupe GSp(4), Astérisque 302, SMF, 2005

[LS98] Martin W. Liebeck and Gary M. Seitz, On the subgroup structure of classical groups, Invent. math. 134 (1998), 427-453

[M04] D. Mauger, Algèbres de Hecke quasi-ordinaires universelles, Ann. Sci. École Norm. Sup. (4) 37 (2004), $171-222$.

[MT02] A. Mokrane, J. Tilouine, Cohomology of Siegel varieties with $p$-adic integral coefficients and applications, in Asterisque 280, 2002

[Pi12] V. Pilloni, Sur la théorie de Hida pour le groupe GSp G $_{2 g}$, Bulletin de la SMF 140 (2012), 335-400

[PiSt] V. Pilloni, B. Stroh, Surconvergence et classicité : le cas déployé, submitted

[P93] R. Pink, Classification of pro- $p$ subgroups of $\mathrm{SL}_{2}$ over a $p$-adic ring, where $p$ is an odd prime, Compositio Math. 88 (1993), 251-264

[P98a] R. Pink, $l$-adic algebraic monodromy groups, cocharacters, and the Mumford-Tate conjecture, J. reine angew. Math. 495 (1998), 187-237

[P98b] R. Pink, Compact subgroups of linear algebraic groups. J. Algebra 206 (1998), 438-504.

[R01] B. Roberts, Global $L$-packets for $\mathrm{GSp}_{2}$ and Theta lifts, Doc. Math. 6 (2001), pp.247-314

[SW01] C. Skinner, A. Wiles, Nearly ordinary deformations of irreducible residual representations, Ann. Fac. Sci. Toulouse Math. (6), 10 (1):185-215, 2001

[Ta93] R. Taylor, On the cohomology of Siegel threefolds, Invent. Math. 114 (1993), pp.289-310

[TU99] J. Tilouine, E. Urban, Several variable $p$-adic families of Siegel-Hilbert cusp eigensystems and their Galois representations, Ann. Sci. E.N.S., 4 série, t. 32, p. 499-574, 1999

[Ti09] J. Tilouine, Cohomologie des variétés de Siegel et représentations galoisiennes associées aux représentations cuspidales cohomologiques de GSp(4), Publ. Math. Besançon, Algèbre et Théorie des Nombres, 2009

[Ur05] E. Urban, Sur les représentations p-adiques associées aux représentations cuspidales de $\mathrm{GSp}_{4}(\mathbb{Q})$, in Formes Automorphes (II), le cas du groupe GSp(4), pp. 151-176, Astérisque 302, SMF, 2005

[Ur11] E. Urban, Eigenvarieties for Reductive Groups, Annals of Math. 174 (2011), 1685-1784

[We05] R. Weissauer, Four-dimensional Galois representations, in Formes Automorphes (II), le cas du groupe GSp(4), pp., Astérisque 302, SMF, 2005

[Y79] H. Yoshida, Weil's representations and Siegel's modular forms. Lectures on harmonic analysis on Lie groups and related topics (Strasbourg, 1979), pp. 319-341, Lectures in Math., 14, Kinokuniya Book Store, Tokyo, 1982.

[Z14] B. Zhao, Local indecomposability of Hilbert modular Galois representations, to appear in Ann. Inst. Fourier (Grenoble) (posted in web: arXiv:1204.4007v1 [math.NT])

Department of Mathematics, UCla, Los Angeles, CA 90095-1555, U.S.A., Université Paris 13, Sorbonne Paris-Cité, LAGA, CNRS (UMR 7539), 99 AV. J.-B. Clément, F-93430, Villetaneuse.

E-mail address: hida@math.ucla.edu, tilouine@math.univ-paris13.fr 